\title{
Role of Imaging in Bariatric Surgery: A Review of the Various Surgical Techniques and Their Complications
}

\author{
Bhavana Girishekar ${ }^{1, \odot ~ S u d a r s h a n ~ R a w a t ~}{ }^{1} \quad$ Rupa Ananthasivan $^{1} \quad$ Pramesh Reddy $^{1}$ Pooja Patil ${ }^{1}$ \\ Kavya Kaushik ${ }^{1}$
}

${ }^{1}$ Department of Radiology, Manipal Hospital, Bengaluru, Karnataka, India

J Gastrointestinal Abdominal Radiol ISGAR 2021;4:109-126.

\author{
Address for correspondence Bhavana Girishekar, MBBS, DNB, \\ Department of Radiology Manipal Hospitals, 98, HAL Old \\ Airport Road, Kodihalli, Bengaluru 560017, Karnataka, India \\ (e-mail: bhavana116@gmail.com). \\ Sudarshan Rawat, MBBS, DMRD, DNB (Radiology), Manipal \\ Hospitals, 98, HAL Old Airport Road, Kodihalli, Bengaluru 560017, \\ Karnataka, India (e-mail: sudarshan.rawat@manipalhospitals.com).
}

\begin{abstract}
Keywords

- bariatric surgery

- gastric banding

- obesity

- roux-en-y gastric bypass

- sleeve gastrectomy

According to the World Health Organization, obesity has reached epidemic proportions globally, with at least 2.8 million people dying each year as a result of being overweight or obese. Bariatric surgery is being increasingly used as a form of treatment, particularly in those patients where lifestyle modifications are deemed insufficient. With the role of radiologists transitioning from a medical to a surgical evaluation in obesity, it is becoming increasingly important to familiarize oneself with the various imaging techniques used in the preoperative and postsurgical evaluation in such cases. This article aims to review the various surgeries performed, their normal imaging appearance, and the various complications that could be encountered.
\end{abstract}

\section{Introduction}

Obesity is an increasingly major health problem worldwide, including in the developing countries like India. Asian Indians are at risk of obesity-related complications at lower levels of body mass index (BMI) when compared with the Caucasians. Specific fat distribution (high truncal and abdominal adiposity) is associated with the influence of insulin sensitivity and other metabolic risk factors. Asian Indians are also more prone to lower glucose metabolism, due to increased insulin resistance and greater procoagulant tendency and dyslipidemia. ${ }^{1}$

The World Health Organization (WHO) definition of overweight and obesity is abnormal or excessive fat accumulation that will impair health. For adults, according to BMI, overweight is defined as BMI greater than or equal to 25 , and obesity as greater than or equal to 30 . For children under 5 years,

published online

March 2, 2021
DOI https://doi.org/

$10.1055 / \mathrm{s}-0041-1725240$ ISSN 2581-9933. overweight and obesity are defined as greater than 2 and 3 standard deviations above the WHO child growth standard median. For children between 5 and 19 years, overweight and obesity are defined as greater than 1 and 2 standard deviations. ${ }^{2}$

India-specific definitions of obesity remain the same as the international classification, but BMI cutoffs for public health action are set at a lower value. BMI ranging from 27.5 to 32.4 are conditionally eligible but not prioritized for surgery. BMI ranging from 32.5 to 34.9 are eligible and conditionally prioritized for surgery, and those greater than or equal to 37.5 are eligible and prioritized for surgery. ${ }^{1}$

Obesity is associated with comorbidities type- 2 diabetes mellitus, hyperlipidemia, hypertension, stroke, metabolic syndrome, ${ }^{3}$ sleep apnea, arthritis, gallstones, nonalcoholic fatty liver disease, depression, polycystic ovarian syndrome, cardiovascular diseases, and cancer. Its health consequences 
range from increased risk of premature death to serious conditions that reduce the general quality of life.

The management of obesity includes lifestyle modifications with particular focus on diet and exercises, pharmacotherapy, ${ }^{4}$ bariatric surgery, and the newer interventional procedure of gastric arterial embolization. According to the fifth registry of the International Federation for the Surgery of Obesity and Metabolic disorders Global registry report of 2019, the total number of surgeries performed in six registered countries was $8,33,687 . .^{5}$ The surgical methods for weight reduction include bypass procedures and restrictive procedures. Bypass procedures act by the process of malabsorption caused by circumventing different lengths of the small bowel. The procedures include jejunoileal bypass, biliopancreatic diversion, and biliopancreatic diversion with duodenal switch. Restrictive procedures act by reducing the gastric volume and thus causing earlier satiety. These are preferred as the risk of malabsorption syndrome is lesser. The procedures include horizontal gastroplasty, vertical banded gastroplasty, adjustable gastric band, and sleeve gastrectomy. Most of the surgical procedures use combined methods for better efficiency and the most commonly performed is laparoscopic Roux-en-Y gastric bypass (RYGB).

The preoperative evaluation of the patient is important and must include a psychological assessment, nutritional education, preparation of a weight loss plan, and medical clearance for surgery. An abdominal ultrasound (US) is important to rule out liver pathology and the presence of cholelithiasis. Patients with acute or chronic cholecystitis or symptomatic gall stones would require a cholecystectomy to be performed at the time of bariatric surgery. Also, these patients would no longer be candidates for endoscopic evaluation of the biliary tract if they develop choledocholithiasis. ${ }^{6}$ An esophagoduodenoscopy is important in those surgical procedures that exclude the stomach, to look for the presence of ulcers, gastroesophageal reflux, or Barrett's esophagus and to rule out malignancy, as the remnant stomach will not be accessible for endoscopic evaluation in the future. ${ }^{7}$

Computed tomography (CT) and magnetic resonance imaging (MRI) can also be used to assess the volumes of visceral adipose tissue (VAT) and subcutaneous adipose tissue (SAT) and measure the densities of each. Many software can be used in the analysis of images obtained by CT and MRI, one of which is the publicly available region growing algorithm by OsiriX. Noncontrast CT or T1- and/or T2-weighted sequence is performed, and the slice acquired at the level of the umbilicus is selected. On CT, fat appears hypodense, with an average attenuation of -45 to- $195 \mathrm{HU}$ while it is bright on $\mathrm{T} 1$ and $\mathrm{T} 2$. On each image, a region of interest (ROI) is drawn over the abdominal wall to delineate the interface between the abdominal wall and visceral fat. The region growing algorithm is then selected, allowing the segmentation ROIs to be drawn in a semiautomated method by pacing the cursor on the visceral fat. The software automatically creates an ROI that includes pixels with gray levels similar to the one selected. At the end of the procedure, the software provides the size of the area included in the region growing algorithm. ${ }^{8}$ Expansion of VAT has a stronger correlation with cardiometabolic risk, while accumulation of SAT has been found to have a more modest association with inflammatory biomarkers. Noninvasive fat density by CT, particularly that of VAT, increases after RYGB and correlates with improved cardiometabolic risk. ${ }^{9}$ All weight loss interventions have however been found to be more effective in reducing SAT, while the percent change of VAT has always been greater than percent change of SAT. ${ }^{10} \mathrm{~A}$ recent meta-analysis ${ }^{11}$ has demonstrated that bariatric surgery leads to greater body weight loss and higher remission rates of type 2 diabetes and cardiometabolic syndrome, compared with nonsurgical treatment of obesity. ${ }^{12}$ The most common adverse events after bariatric surgery were iron deficiency anemia (malabsorptive bariatric surgery) and reoperations. ${ }^{11}$

The imaging methods available in the postoperative evaluation in bariatric surgeries include conventional radiography (which is highly nonspecific and seldom performed), upper gastrointestinal (GI) contrast studies, endoscopy, and CT. The protocol for upper GI contrast studies includes an initial abdominal scout radiograph followed by the ingestion of 50 to $100 \mathrm{~mL}$ of dilute water-soluble contrast diatrizoate meglumine and diatrizoate sodium (Gastrograffin, Bracco Diagnostics), and in the absence of a leak, high-density barium can be used for better luminal opacification. The contrast column is followed until the distal small bowel (particularly in cases of RYGB). Barium is not advisable for leak testing, as it can cause chemical peritonitis. In cases that are equivocal on upper GI contrast studies, CT is the investigation of choice, as small leaks are better visualized. Similar dilute water-soluble contrast is used here as well. In fact, in a substantial proportion of patients, even CT does not demonstrate extravasation of contrast and a collection is usually the only sign ( - Table $\mathbf{1}$ ).

Table 1 Summary outlining the major differences in the commonly performed bariatric surgeries

\begin{tabular}{|l|l|l|l|}
\hline & Laparoscopic SG & RYGB & $\begin{array}{l}\text { Laparoscopic adjustable } \\
\text { gastric band }\end{array}$ \\
\hline Surgical techniques & $\begin{array}{l}\text { Creation of a tubular gastric } \\
\text { pouch by exclusion of major- } \\
\text { ity of the gastric volume }\end{array}$ & $\begin{array}{l}\text { Formation of a gastric pouch anas- } \\
\text { tomosed with the jejunum; bowel } \\
\text { continuity maintained with a distal } \\
\text { jejunojejunal anastomosis }\end{array}$ & $\begin{array}{l}\text { Silicone band around the proximal } \\
\text { stomach to create a pouch with a } \\
\text { narrow stoma }\end{array}$ \\
\hline Method of weight loss & Restrictive & Restrictive + bypass & Restrictive \\
\hline Common complications & Staple line hemorrhage/leak & $\begin{array}{l}\text { Anastomotic stricture/leak, small } \\
\text { bowel obstruction }\end{array}$ & Stomal stenosis, band slippage \\
\hline
\end{tabular}

Abbreviations: RYGB, Roux-en-Y gastric bypass; SG, sleeve gastrectomy. 
This article aims to review the normal radiological anatomy postbariatric surgery and the various possible complications and their imaging features.

\section{Laparoscopic Sleeve Gastrectomy}

This is a relatively new surgical procedure which was started in the year 1999 and has become increasingly popular since with a large number of countries preferring this over RYGB. This procedure is a modification of the Magenstrasse and Mill procedure ${ }^{13}$ and includes the creation of a long narrow gastric pouch by exclusion of approximately $75 \%$ of the gastric volume, thereby facilitating early satiety. In addition, there is reduced production of the hormone ghrelin (produced in the gastric fundus), which is known to be an appetite stimulant. It is performed by dividing the stomach along the long axis and resecting the greater curvature of the fundus, body and proximal antrum, thus making it irreversible. ${ }^{14}$

\section{Normal Anatomy}

On upper GI contrast study, a long tubular gastric pouch is noted, which could have a slightly widened distal end (due to preserved pylorus). CT scan is generally performed only in case of a suspicious complication, in which the normal gastric pouch is seen as a tubular structure adjacent to the surgical staples along the greater curvature of the remnant stomach, with abundant mesenteric fat in the expected location of the stomach (-Figs. 1-3).

\section{Complications}

The incidence of complications in postoperative cases of sleeve gastrectomy is $7.4 \%{ }^{16}$ They are as follows:

1. Staple line hemorrhage: This can be intraluminal (diagnosed and treated by endoscopy) or intrabdominal without upper GI bleed. Conventional radiography is nonspecific for hemorrhage and this would require a CT scan either with or without intravenous (IV) contrast to look for the presence of hematoma and active contrast extravasation. This is generally seen within the first few hours postsurgery (-Fig. 4).

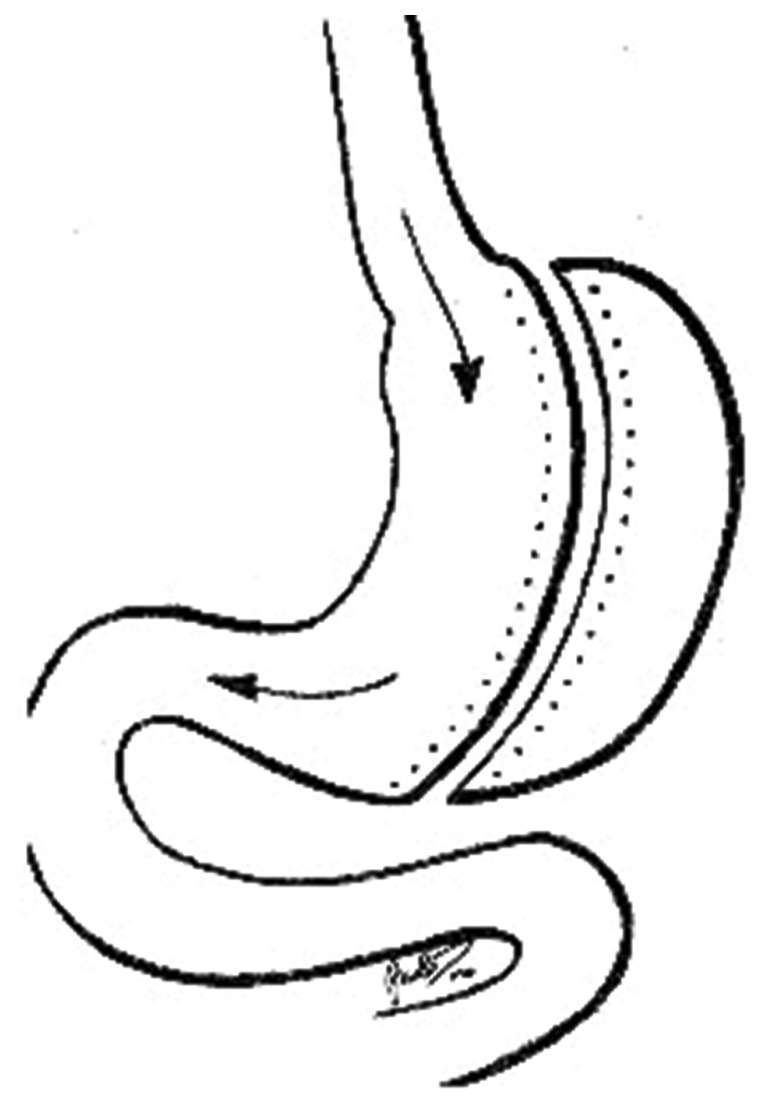

Fig. 1 Schematic representation of creation of a gastric pouch by the exclusion of $75 \%$ of the gastric volume. ${ }^{15}$
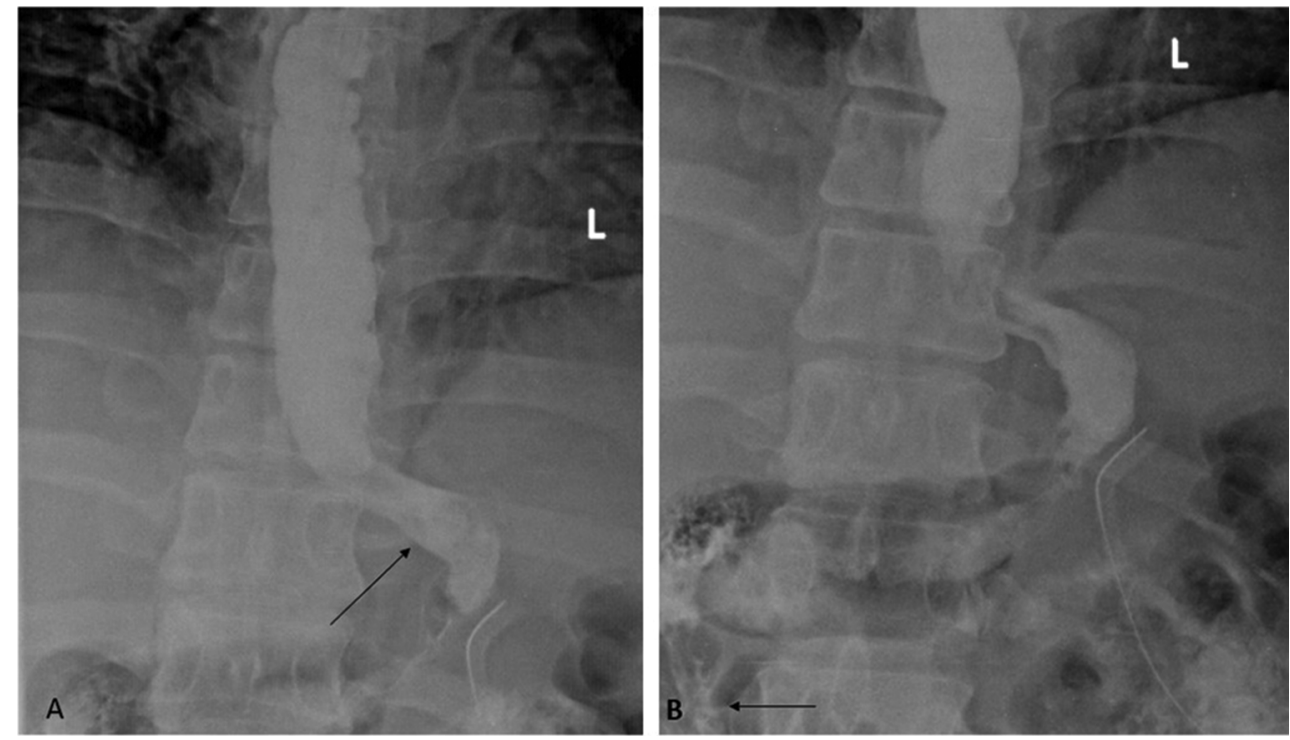

Fig. 2 Normal appearance of sleeve gastrectomy on upper gastrointestinal study showing the narrow gastric pouch (line arrow in A) and a slightly delayed film showing contrast in the duodenum (line arrow in B). 

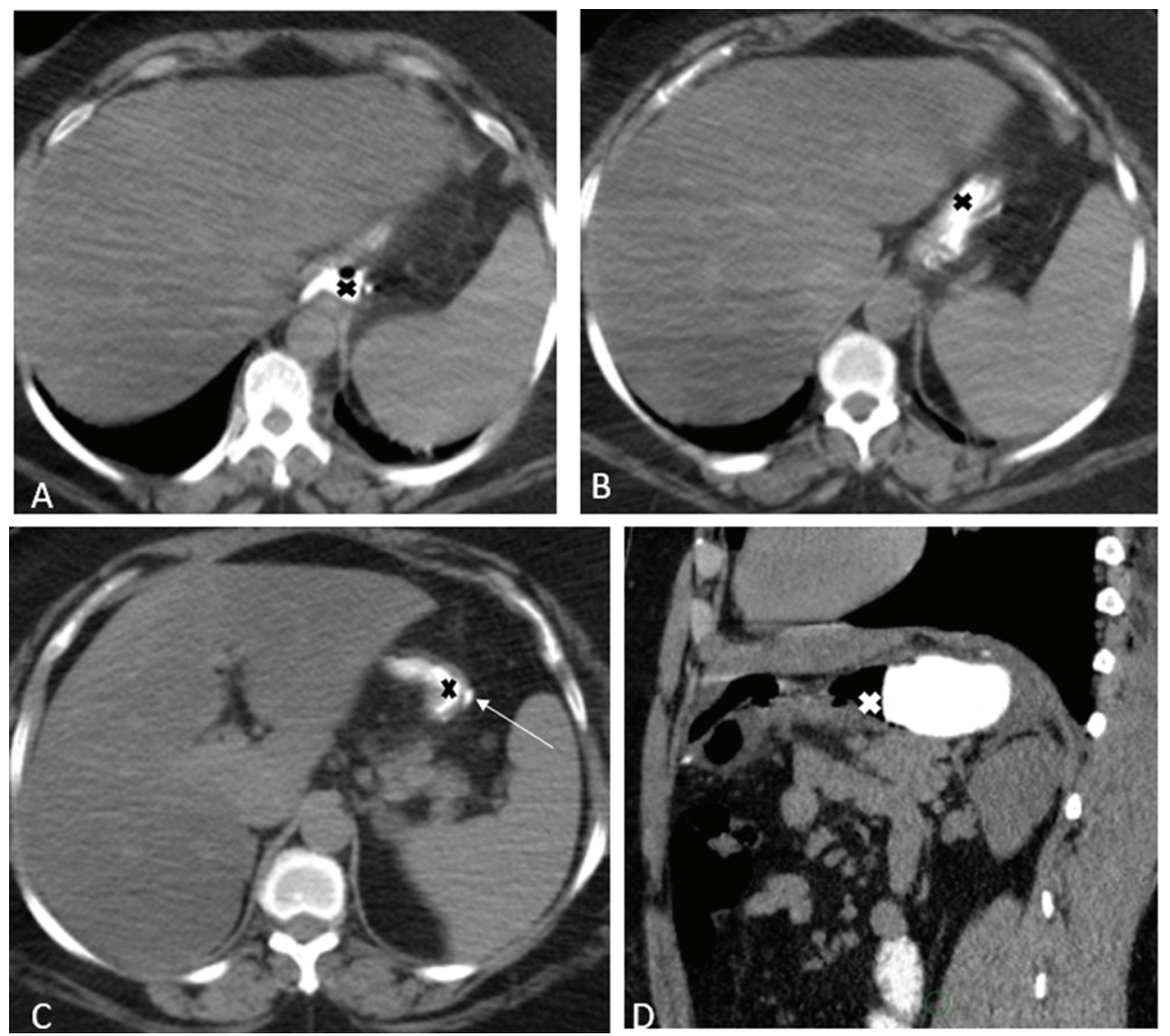

Fig. 3 Normal appearance of sleeve gastrectomy on computed tomography (CT). Axial (A-C), sagittal (D) CT showing the staple line along the greater curvature of the stomach (line arrow in $\mathrm{C}$ ) with the narrowed gastric pouch (X).
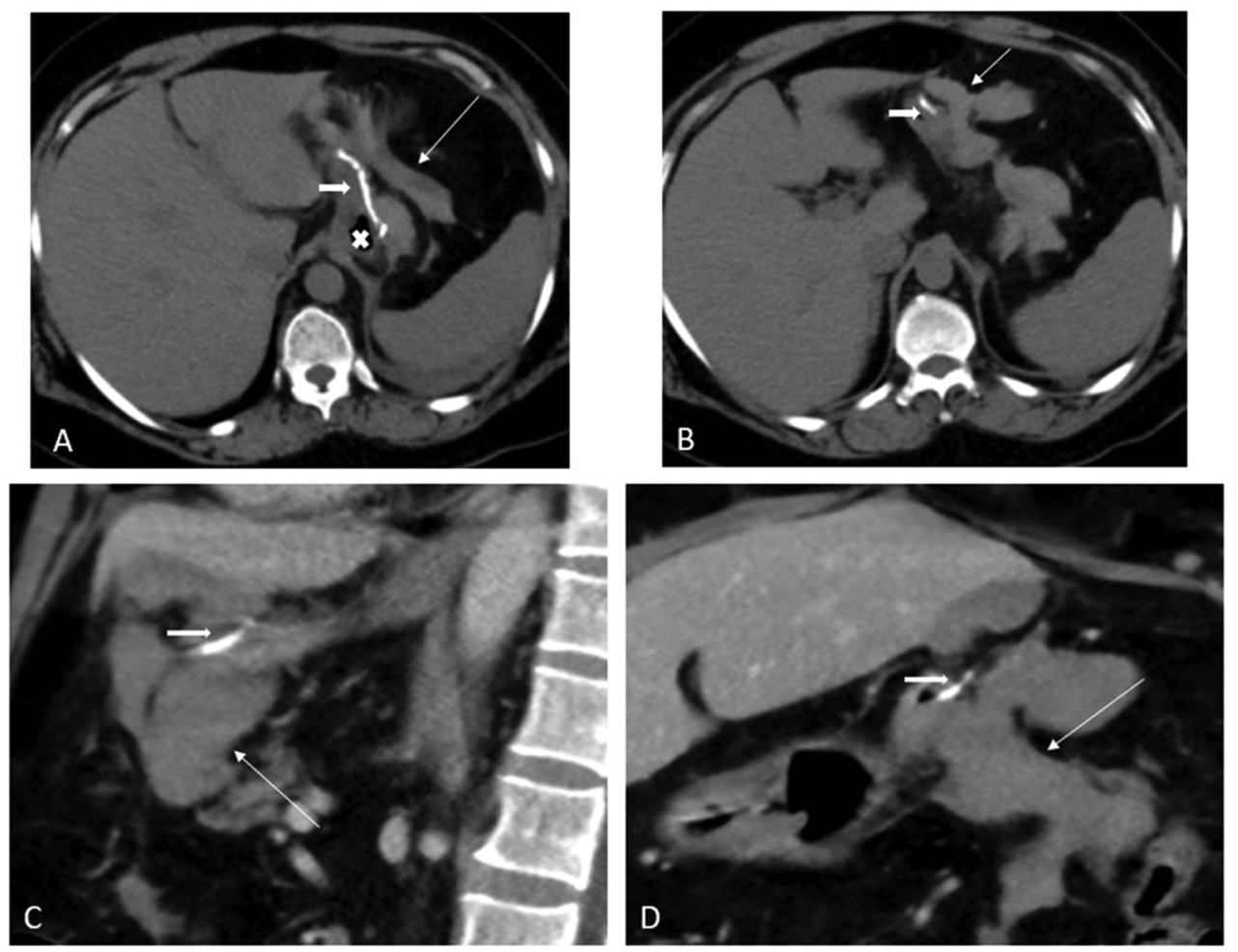

Fig. 4 Axial noncontrast (A, B), contrast-enhanced sagittal (C) and coronal (D) computed tomography in a patient with abdominal pain one day postsleeve gastrectomy showing the staple line along the greater curvature of the stomach (block arrows in all) with the narrow gastric pouch (marked X in A). Line arrows show lobulated hematoma inferolateral to the staple line, attenuation of 55 to $65 \mathrm{HU}$ on the plain scan and remaining unenhanced on the postcontrast scan. 
2. Staple line leak: It can occur early (within the first 3 days of surgery), intermediate (within the first week), or late (more than 8 days later). It usually occurs from the superior aspect of the staple line, and the presence of extraluminal oral contrast on upper GI study with a collection or into the peritoneal cavity is diagnostic. CT scans can be used for subtle leaks and small abscesses (-Figs. 5-7).
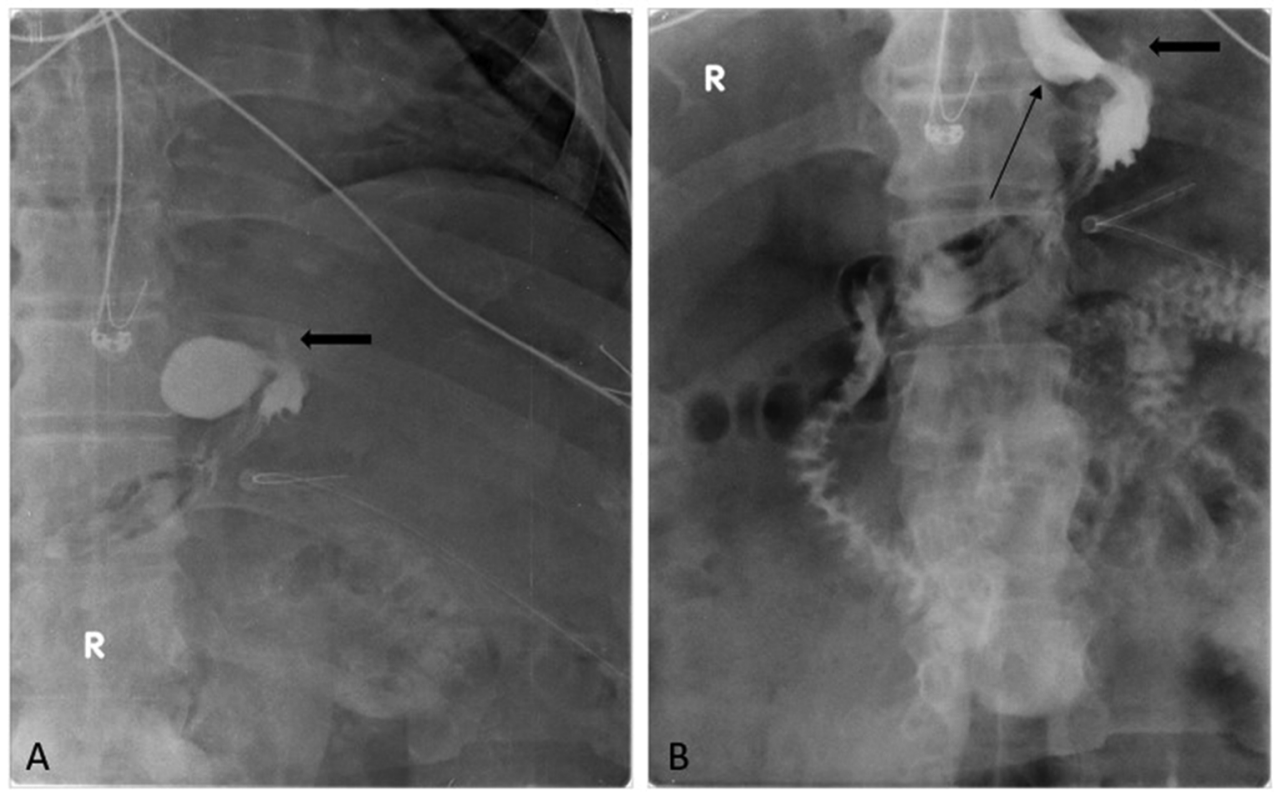

Fig. 5 Upper gastrointestinal study in a patient 2 days postsleeve gastrectomy showing extraluminal contrast (block arrow in $\mathbf{A}$ and $\mathbf{B}$ ) leaking along the superior aspect of the gastric pouch (line arrow in B).
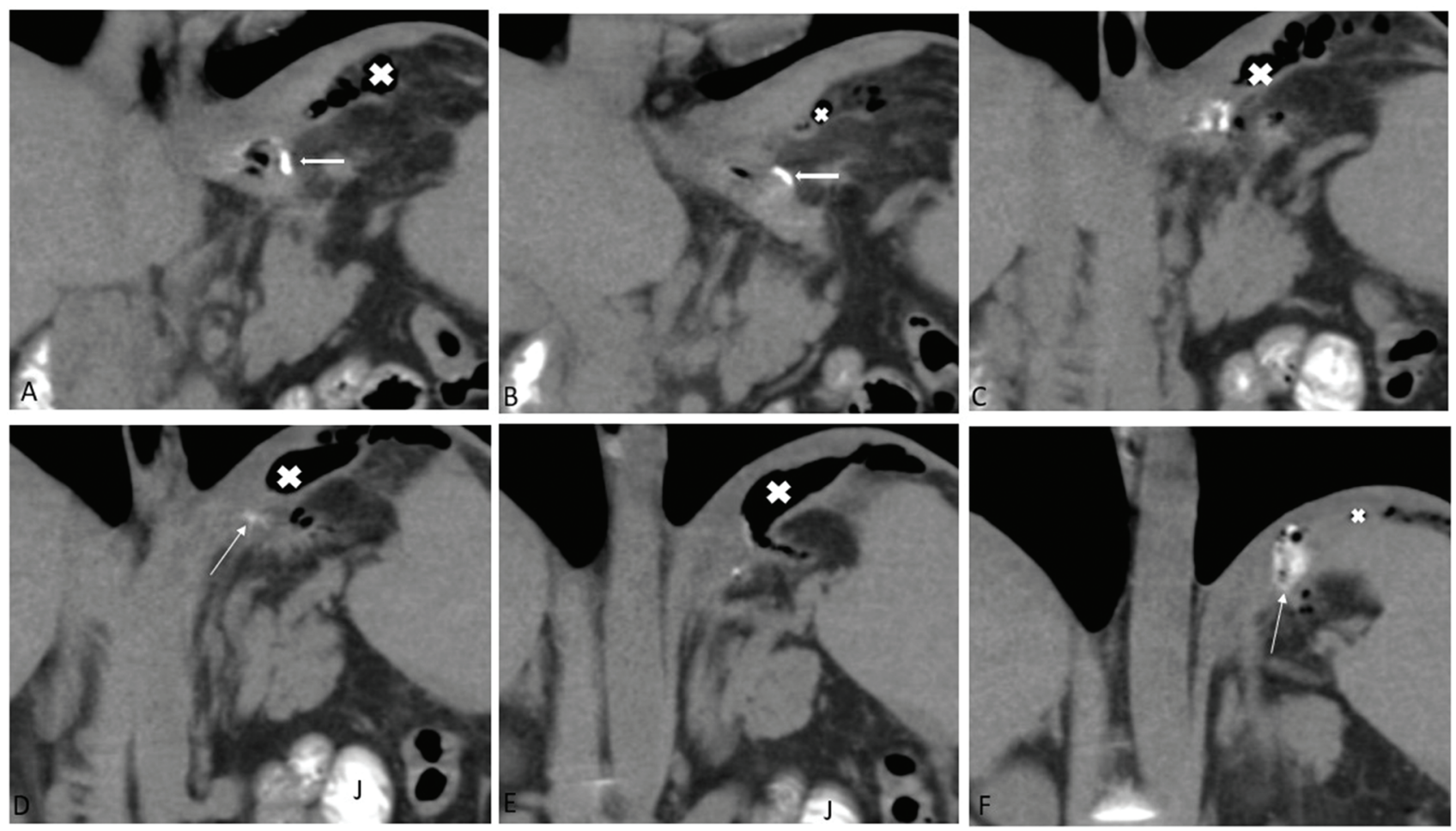

Fig. 6 Coronal noncontrast computed tomography (A-F from anterior to posterior) in a patient 2 days postsleeve gastrectomy showing staple line (block arrow in $\mathbf{A}$ and $\mathbf{B}$ ) along the greater curvature of the stomach, contrast-opacified jejunal loops (marked J in $\mathbf{D}$ and $\mathbf{E}$ ). Air pockets and fluid collection (marked $\mathrm{X}$ in all) noted beneath the left dome of the diaphragm with leak of orally administered contrast (line arrow in $\mathbf{D}$ and $\mathbf{F}$ ). 

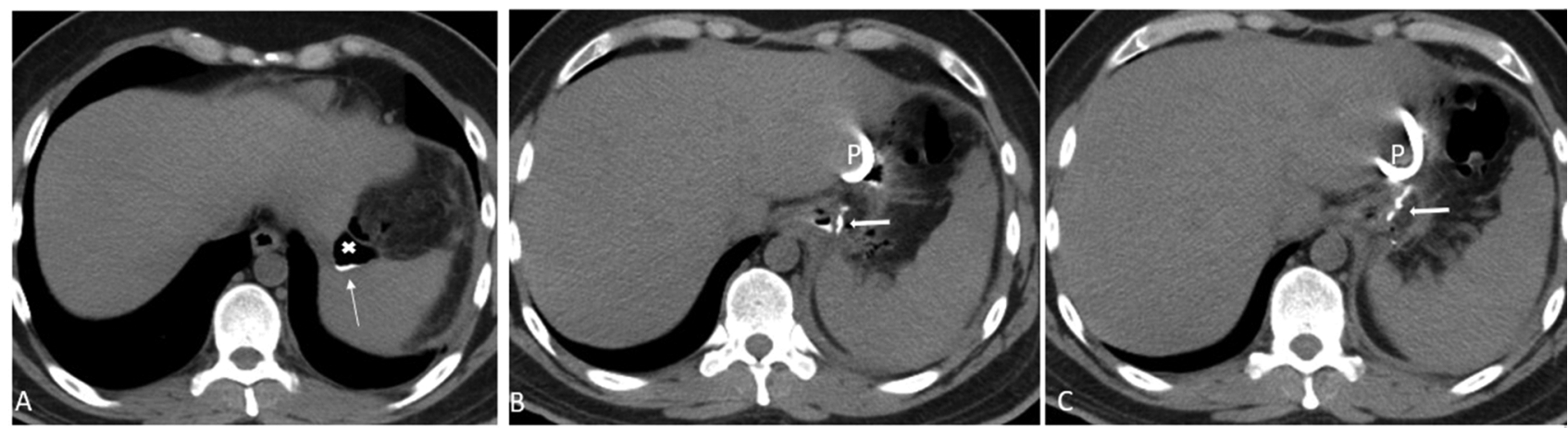

Fig. 7 Axial noncontrast computed tomography (A-C) in the same patient (as in - Fig. 6) from cranial to caudal showing staple line (block arrow in $\mathbf{B}$ and $\mathbf{C}$ ). The cranial most image $(\mathbf{A})$ shows extraluminal contrast (line arrow) within the collection (marked X). Therapeutic pig-tail catheter (marked $\mathbf{P}$ in $\mathbf{B}$ and $\mathbf{C}$ ) was introduced under image guidance.

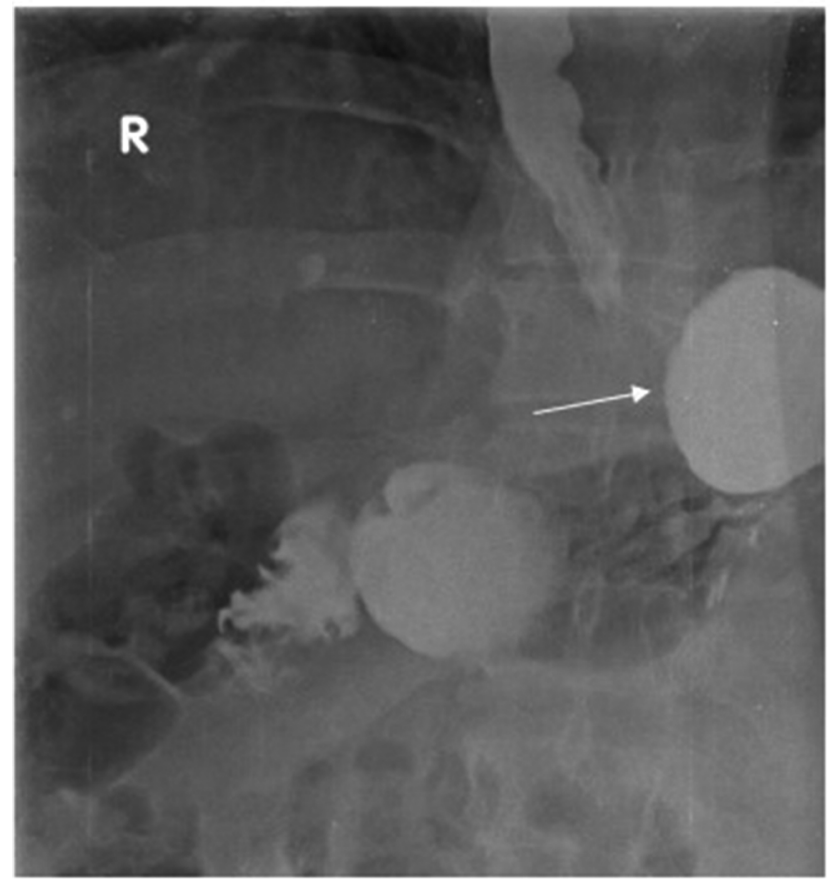

Fig. 8 Upper gastrointestinal study in a patient who presented with recurrent weight gain 3 years after sleeve gastrectomy, showing a dilated gastric pouch (line arrow).

4. Gastric dilatation: This might occur in some patients who present with inadequate weight loss or recurrent weight gain, requiring revision surgery. The sleeve on upper GI study no longer has a tubular appearance and is widened ( - Fig. 8 ).

5. Gastroesophageal reflux: This occurs due to reduced gastric compliance, distorted gastric anatomy, and stasis. On upper GI study, the reflux of oral contrast is seen into the dilated esophagus along with features of esophageal dysmotility.

6. Rarer complications include splenic infarction due to injury to splenic arterial branches (identified as a wedge-shaped non-enhancing hypodense area in the periphery of the spleen on $\mathrm{CT}$ ), portomesenteric vein thrombosis causing bowel wall ischemia (seen as dilated bowel loops with wall thickening, pneumatosis intestinalis and sometimes gangrenous bowel resulting in perforation), and symptomatic cholelithiasis.

\section{Laparoscopic Roux-En-Y Gastric Bypass}

This was first performed laparoscopically by Wittgrove et al in $1994^{17}$ and used to be the most popular bariatric surgery with the highest long-term success and most sustained weight loss. ${ }^{18-21}$

Technique: It involves dividing the stomach to form a small, proximal gastric pouch completely separated from the larger lower stomach called the gastric remnant. This is followed by gastrojejunostomy to create a contiguous alimentary tract. The alimentary (Roux limb) and biliopancreatic limbs of the Roux-en-Y are then constructed. The jejunum is divided approximately $50 \mathrm{~cm}$ distal to the ligament of Treitz to create the biliopancreatic limb. The jejunum is then measured between approximately 60 and $100 \mathrm{~cm}$ based on the BMI of the patient $(60 \mathrm{~cm}$ for $<50$, and $100 \mathrm{~cm}$ for $>50)^{7}$ distal to this division for the site of the end-to-side jejunojejunostomy connecting the biliopancreatic and Roux limbs. The Roux limb is then brought up to the stomach by dividing the greater omentum at the inferior margin of the transverse colon. Another technique is the retrocolic/retrogastric approach, which is associated with a higher rate of complications..$^{22}$ This is used when the division of the omentum is insufficient to facilitate a tension-free alimentary limb. The Roux limb is brought up posterior to the transverse colon and stomach through a surgically created window in the transverse mesocolon. Petersen's space between the Roux limb and the transverse colon mesentery is closed to prevent future small bowel herniation. The most common site for herniation was found to be the retrocolic mesenteric defect, which led to small bowel obstruction. ${ }^{23}$ Primary closure of the mesenteric defects reduces the incidence of internal hernia but can lead to other potential complications like mesenteric hematoma with circulatory impairment $^{24}(-$ Fig. 9).

\section{Normal Anatomy}

On upper GI contrast study, the gastric pouch is small and measures $4 \mathrm{~cm}$, with an approximate volume of 15 to $20 \mathrm{~mL}$, 
although this may vary. The gastrojejunal anastomosis is visualized in profile in the frontal views in most cases, except when the jejunum is connected to the anterior or posterior aspect of the pouch, in which case, steep oblique/lateral views are to be obtained. A small outpouching called "rabbit ear" representing the end-to-side gastrojejunal anastomosis can sometimes be seen as a normal variant. ${ }^{25}$ Bowel signature can be seen, as this represents the efferent loop, and it will not progressively increase in size; this helps differentiate it from a leak. The head of the contrast column in the alimentary limb is to be followed until the jejunojejunal anastomosis is visualized up to the terminal ileum. Narrowing

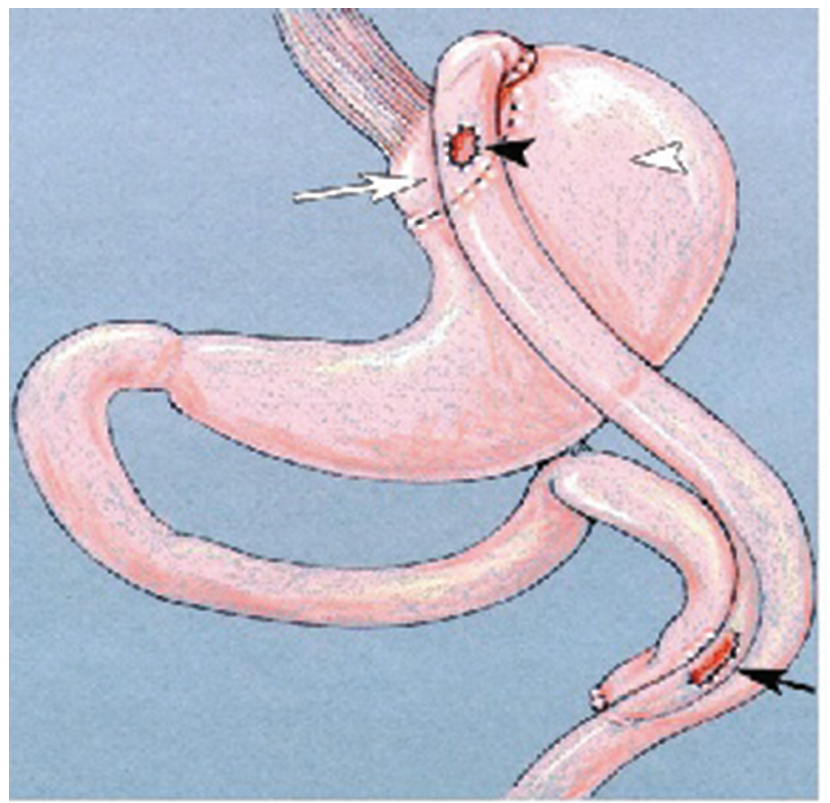

Fig. 9 Normal surgical anatomy after Roux-en-Y gastric bypass with the white arrow showing the gastric pouch, which was excluded from the larger stomach (white arrowhead). Black arrowhead shows the gastrojejunal anastomosis, and black arrow shows the jejunojenunal anastomosis. ${ }^{14}$ of the alimentary limb at the surgical opening of the transverse mesocolon in the retrocolic/retrogastric approach can be confused with an ischemic stricture or jejunal narrowing (-Fig. 10).

On CT, the high attenuation suture line of the gastrojejunal anastomosis is usually seen in the epigastrium, and the distal jejunojenunal anastomotic site is lower in the abdomen. The orally administered contrast can be seen opacifying the gastric pouch, across the gastrojejunal anastomosis into the alimentary limb, and moving across the jejunojejunal anastomosis into the distal small bowel. CT has the added advantage of being able to assess the excluded stomach and the biliopancreatic limb, which are not opacified with oral contrast. These should not be confused with fluid collections or abscesses. Small bowel obstruction and internal hernia are better demonstrated on CT ( - Fig. 11).

\section{Complications}

1. Anastomotic leak: This is one of the most feared complications and presents with pain, fever, tachycardia, nausea and vomiting, reduced urine output, and hypotension. ${ }^{26}$ It happens within the first 10 days postsurgery and usually occurs at the gastrojejunostomy site. The other possible sites include the gastric pouch, blind jejunal stump, excluded stomach, and the jejunojejunal anastomosis. On upper GI study, gastrojejunal site leaks are best demonstrated in the supine or left posterior oblique positions, are seen as blind-ending tracks or sealed off collections, and less frequently as free leaks into the peritoneal cavity. They can sometimes extend to the left upper quadrant as subphrenic collections. CT scan is superior in demonstrating the presence of abscesses and subtle leaks (-Figs. 12-13).

2. Anastomotic stricture: Early postoperative edema can lead to stenosis at the gastrojejunal anastomosis which resolves within the first week. ${ }^{27}$ Fixed strictures usually
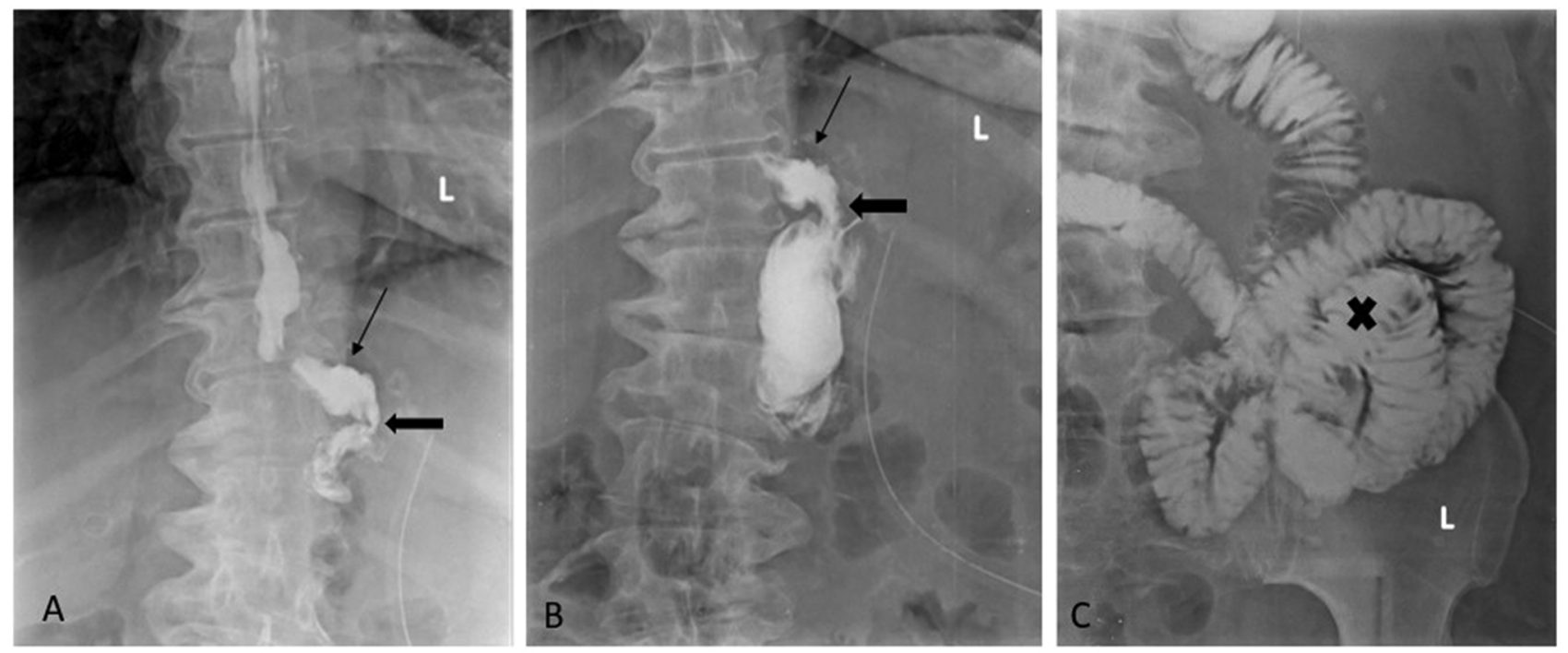

Fig. 10 Normal appearance of Roux-en-Y gastric bypass on upper gastrointestinal study $(\mathbf{A}-\mathbf{C})$ showing narrow gastric pouch (line arrow in $\mathbf{A}$ and $\mathbf{B}$ ), and gastrojejunal anastomosis (black arrow in $\mathbf{A}$ and $\mathbf{B}$ ) with the contrast-opacified jejunal loops (marked $\mathbf{X}$ in $\mathbf{C}$ ) in the delayed image. 

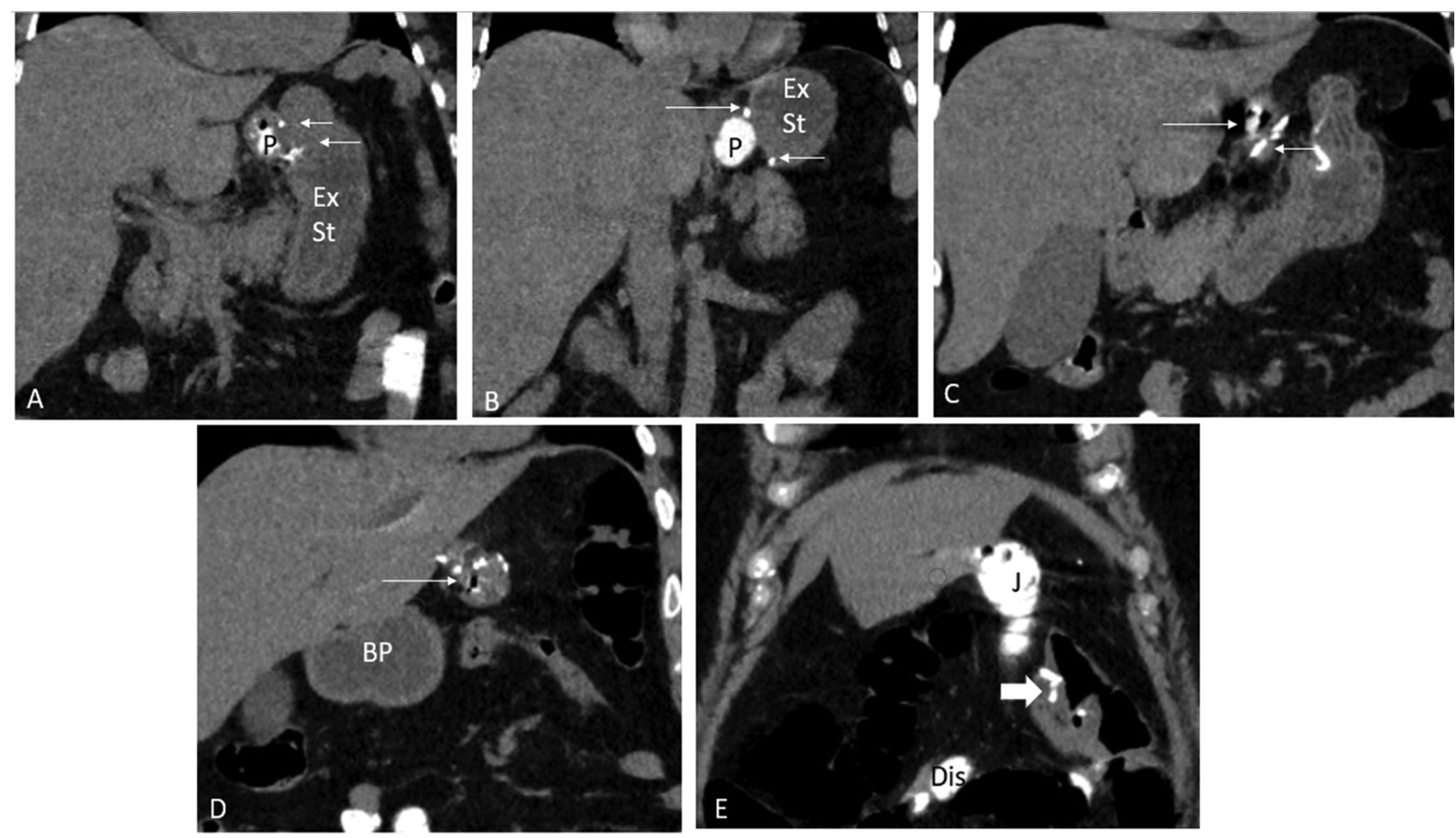

Fig. 11 Normal appearance of Roux-en-Y gastric bypass on coronal noncontrast computed tomography (A-E from posterior to anterior) showing contrast opacified gastric pouch (marked $\mathrm{P}$ in $\mathbf{A}$ and $\mathbf{B}$ ), jejunum (marked $\mathrm{J}$ in $\mathbf{E}$ ) and distal small bowel (marked D is in E), with nonopacified excluded stomach (marked Ex St in $\mathbf{A}$ and $\mathbf{B}$ ) and biliopancreatic limb (marked BP in $\mathbf{D}$ ). The gastrojejunal (line arrows in $\mathbf{A}-\mathbf{D}$ ) and jejunojejunal anastomosis (block arrow in $\mathbf{E}$ ) are also seen.
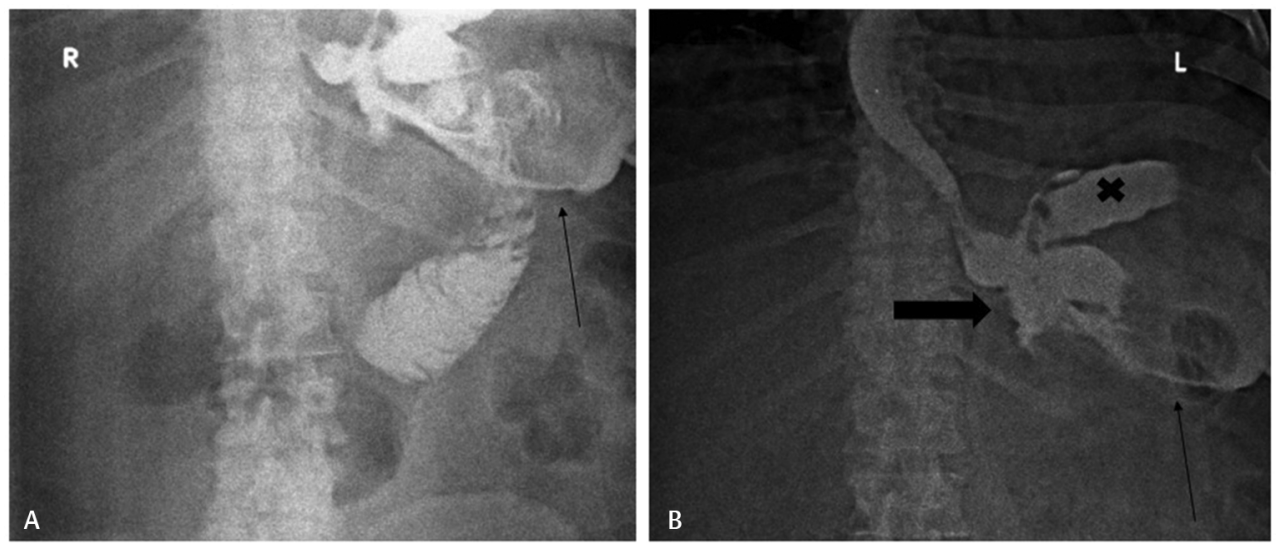

Fig. 12 Upper gastrointestinal study (A and $\mathbf{B}$ ) in a patient 8 two days postRoux-en-Y gastric bypass showing extraluminal leak of contrast (line arrows) with passage of contrast across the gastrojejunal anastomosis (block arrow in B). Another pocket of contrast seen along the superolateral aspect (marked X in $\mathbf{B}$ ) was confirmed to be a contrast leak below the left dome of the diaphragm on the subsequent computed tomography.

occur in 3 to $9 \%$ of patients due to ischemia and scarring and can be detected on upper GI studies by the presence of smooth narrowing at the anastomotic site, with a dilated gastric pouch and delayed gastric emptying. Strictures with an anterior or posterior location in relation to the anastomosis would require a steep oblique or lateral view, while those inferior to the anastomosis can be visualized in the frontal view..$^{14}$ They can also be diagnosed on endoscopy and treated with dilatation. ${ }^{28}$ Less commonly, stenosis can occur at the jejunojejunal anastomosis and causes small bowel obstruction, which is better evaluated on CT (-Fig. 14).
3. Small bowel obstruction (SBO): This can occur in up to $5 \%$ of patients and may be due to adhesions, internal hernias, abdominal wall hernias, strictures, bezoar or intussusception at the jejunojejunal anastomosis. The $\mathrm{ABC}$ classification of internal hernias includes the following 29 :

A. Type $\underline{\mathbf{A}}$ : Dilatation of the $\underline{\mathbf{A}}$ limentary limb with decompressed biliopancreatic limb and distal common channel. This is seen on upper GI studies as dilated Roux limb at or proximal to the jejunojejunal anastomosis.

B. Type B: Dilatation of the Biliopancreatic limb only, with decompressed alimentary limb and distal common channel. This 

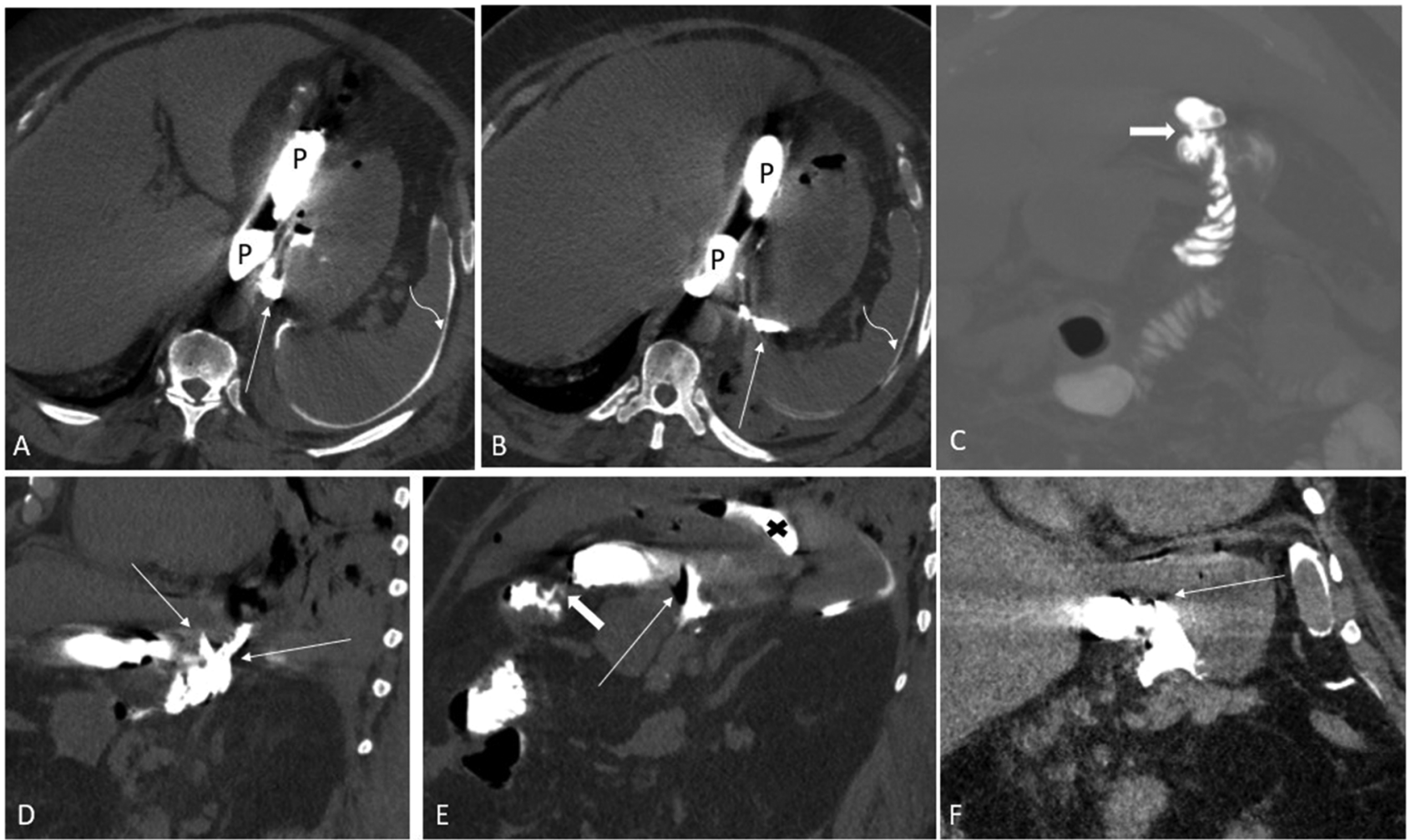

Fig. 13 Axial noncontrast computed tomography (A and B) in the same patient as in - Fig. 12 showing extraluminal contrast (line arrows) outside the gastric pouch (marked $\mathrm{P}$ ) and along the spleen (curved arrows). Coronal maximum intensity projection image (C) shows the gastrojejunal anastomosis (block arrow) more anteriorly. Sagittal image (D and E) showing extraluminal contrast posterior and inferior to the gastric pouch (line arrows) and below the left dome of the diaphragm (marked X) with the gastrojejunal anastomosis more anteriorly (block arrow). Coronal image (F) also showing large area of leak of contrast (line arrow).
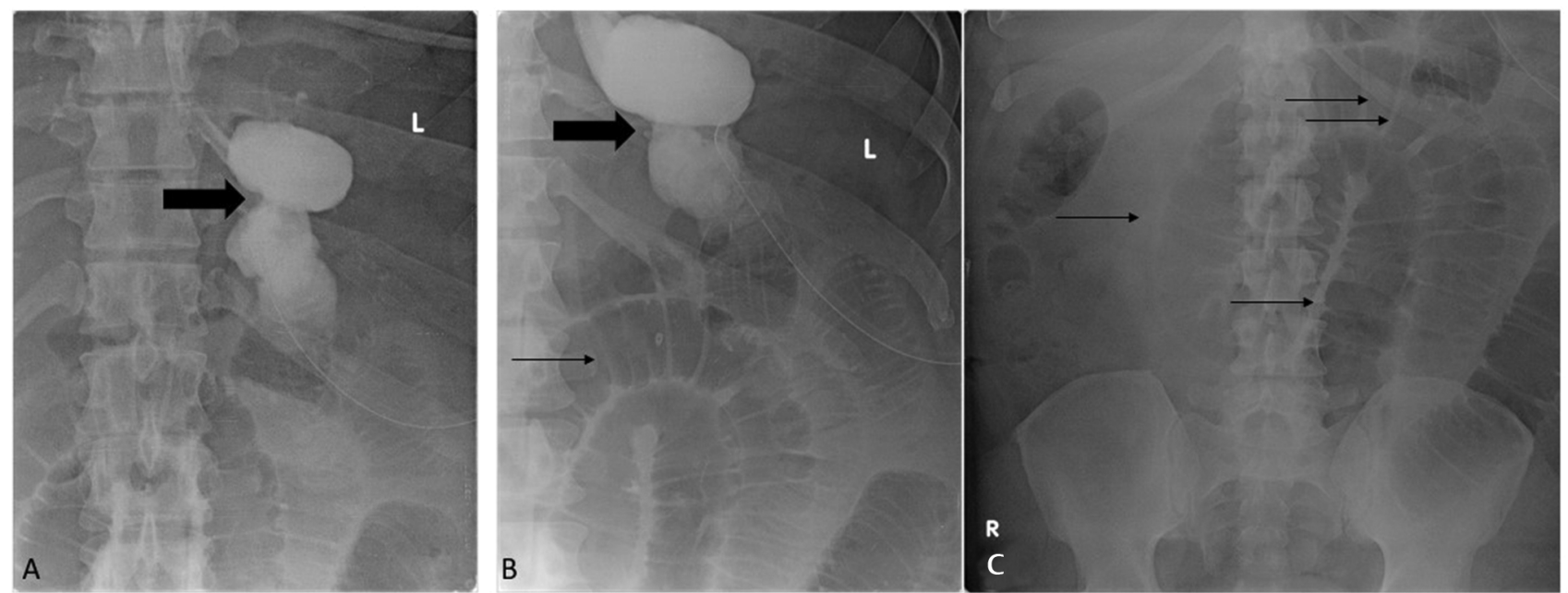

Fig. 14 Upper gastrointestinal study (A, B followed by a delayed film-C) in a patient 8 days post Roux-en-Y gastric bypass showing smooth passage of contrast across the gastrojejunal anastomosis (block arrow in $\mathbf{A}$ and $\mathbf{B}$ ) with holdup of contrast. The delayed film showed no contrast opacification of the distal small bowel loops with dilated jejunal loops (line arrow in B and $\mathbf{C}$ ) due to stricture at the jejunojejunal anastomosis.

is a closed-loop obstruction and can lead to perforation of the excluded stomach and is usually diagnosed on CT, as the biliopancreatic limb remains unopacified on upper GI studies.

C. Type $\underline{\mathrm{C}}$ : Obstruction of the distal $\underline{\text { Common channel distal }}$ to the jejunojejunostomy with dilatation of both the alimentary and the biliopancreatic limbs.
SBO due to adhesions is more common with open rather than laparoscopic surgeries, while internal hernia (IH) results more commonly from the laparoscopic approach. Adhesions and scarring due to peritoneal irritation that result from an open surgery prevent the herniation of bowel loops. ${ }^{30}$

4. Internal hernias: They present with small bowel obstruction, usually postlaparoscopic surgery, with an incidence 
of approximately $3 \%$. The bowel loops can herniate either through a defect in the transverse mesocolon (created for the Roux limb in retrocolic/retrogastric approach), mesenteric defect adjacent to jejunojenjunal anastomosis, or Peterson's defect posterior to the Roux limb, between the caudal surface of the transverse mesocolon and the Roux limb mesentery.

Contrast studies for these would include a small bowel follow-through (SBFT) examination in all suspected cases. Clustering of bowel loops, most commonly in the left and/ or midabdomen displacing the rest of the bowel loops, displaced jejunojejunal anastomotic line, change in bowel configuration (compared with the prior scan), stasis of contrast in the clustered loops and entry and exit of the bowel loops through the hernial orifice can be seen. Dilated obstructed bowel can be seen in some cases but is not always present. ${ }^{31}$

CT scan is more sensitive and specific in the diagnosis of internal hernia and shows the following signs: swirled mesenteric root fat and vessels, clustered small bowel loops with dilatation, mushroom-shape of the herniated mesenteric root, with crowding and stretching of the mesenteric vessels. The relatively lesser described signs include the presence of small bowel loops other than the duodenum passing posterior to the superior mesenteric artery (SMA), tubular distal mesenteric fat surrounded by bowel (i.e., hurricane eye sign), beaking of the superior mesenteric vein, ${ }^{32}$ and right-sided displacement of the jejunojejunal anastomosis. Of these, the swirled mesentery is the best single predictor of internal hernia, ${ }^{33}$ caused by a rotation of the complete mesentery, which can be recognized by following the routes of the mesenteric vessels (-Figs. 15-17).

5. Intussusception: They typically occur at or near the jejunojejunal anastomosis, and the staple line acts as a lead point. It is usually transient and nonobstructing; if persistent and large, they can cause SBO.

6. Ulcers: Marginal ulcers can occur at the gastrojejunal anastomosis and is hypothesized to be due to chronic exposure of the mucosa to acid entering the alimentary limb. It can also be due to suture material irritation, configuration of the pouch, tension and ischemia of the alimentary limb. They can present with upper GI bleed and are diagnosed on endoscopy. On upper GI studies, it is seen as a niche at the anastomotic site.

7. Small bowel (jejunal) ischemia: This occurs due to tension on the surgically mobilized jejunum, which compromises its vascular supply. Upper GI study reveals thickened, spiculated folds or thumb printing. $\mathrm{CT}$ is more specific and reveals wall thickening with mesenteric root edema and engorged vessels. In the case of bowel infarction, altered enhancement of the bowel wall, pneumatosis and frank perforation can occur. Chronic ischemia can lead to the development of stricture, with tubular narrowing of the bowel lumen showing smooth, tapered margins along with obliterated folds that can be diagnosed on upper GI studies or CT.
8. Recurrent weight gain: This can occur due to the presence of the gastrogastric fistula (GGF), with a dehiscent staple line causing communication of the gastric pouch and the excluded stomach. These can be diagnosed as an anastomotic leak on upper GI study with the accumulation of contrast (as compared with free flow into the peritoneal cavity), and contrast accumulation in the excluded stomach and not in the biliopancreatic and duodenum on CT. In the past, it has been suggested that small leaks into the excluded stomach are of little clinical importance, while others have found that even small leaks may be associated with recurrent weight gain, necessitating surgical revision. ${ }^{34}$

\section{Laparoscopic Adjustable Gastric Banding}

The laparoscopic approach to gastric banding (1986) was developed in 1996, following which the adjustable version was made available in 2001. It is a reversible procedure that is technically simpler and associated with a similar incidence of postoperative morbidity as the other procedures. However, recent studies have shown a higher incidence of revision procedures due to complications or inadequate weight loss. ${ }^{35-37}$

The procedure includes placement of a silicone band (sutured to the stomach) around the proximal stomach, creating a pouch with a narrow stoma through the band and communicating with the rest of the stomach. This is done to restrict the passage of the food. The inner aspect of the band has an inflatable balloon cuff that is connected by a tube to a subcutaneous port placed in the anterior abdominal wall. Fluid is added or removed from the port to inflate or deflate the cuff, based on the patient's weight loss curve ( - Fig. 18).

\section{Normal Anatomy}

The band is radiodense and is placed obliquely (the lateral side above the medial side) in the left epigastric region, the horizontal axis of which makes an angle of approximately 4 to 58 degrees with the spinal column. ${ }^{36}$ Discontinuity or kinking of the tubing and the inflatable cuff is to be observed on the scout view. On oral administration of contrast, gastric pouch ( $4 \mathrm{~cm}$ diameter) is seen above the band with tapered narrowing, traversing the stoma which normally measures 3 to $5 \mathrm{~mm} \cdot{ }^{39,40}$ Frontal or slightly right posterior views are taken to prevent the stoma from being obscured by the opacified fundus of the stomach. On CT, the radio-opaque band is seen with the tubing traversing the peritoneal space and rectus muscles to the subcutaneous port along the anterior rectus sheath ( - Fig. 19).

Complications: Early postoperative complications include gastric perforation $(<0.5 \%)$, early band slippage $(<0.15)$, and acute stomal stenosis $(<0.4 \%){ }^{41,42}$

1. Stomal stenosis: This the most common complication and occurs when the band is too tight, presenting with nausea and vomiting, regurgitation and dysphagia. Upper GI study reveals excessive narrowing of the stoma, with dilatation of the proximal stomach, and pouch-esophageal reflux with slow emptying into the remaining stomach. 

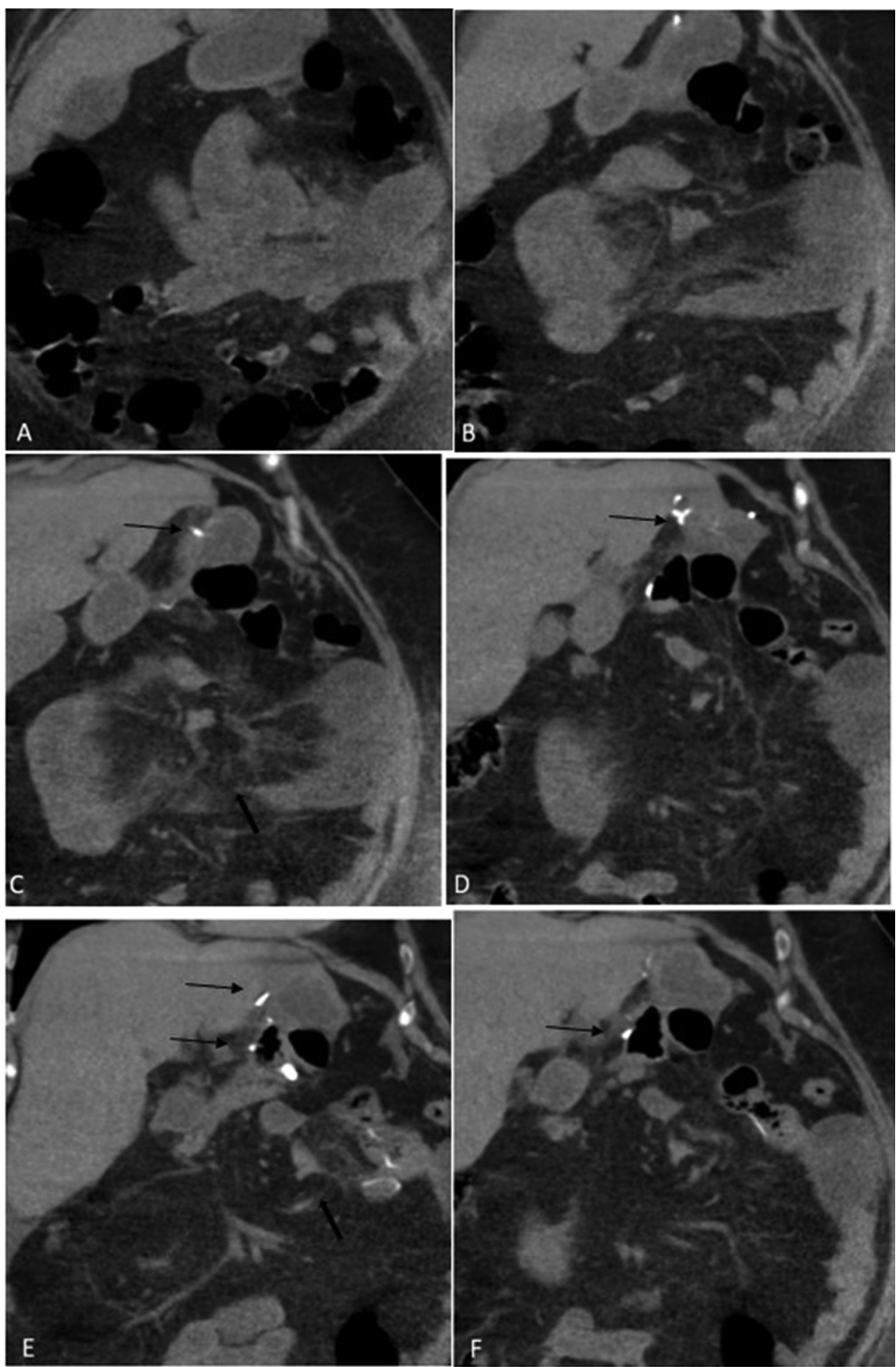

Fig. 15 Coronal contrast-enhanced computed tomography (A-F from anterior to posterior) in a patient 8 days post Roux-en-Y gastric bypass showing gastrojejunal anastomosis (line arrows in $\mathbf{C}-\mathbf{F}$ ) and jejunojejunal anastomosis (in $\mathbf{E}$ and $\mathbf{F}$ ). Clumped dilated small bowel loops (marked $\mathbf{X}$ in $\mathbf{A}-\mathbf{F}$ ) are seen with swirling of the mesenteric vessels (in $\mathbf{E}$ ) and radiating mesenteric vessels (in $\mathbf{C}$ ) marked with block arrows suggestive of internal hernia. This was confirmed to be Peterson's hernia intraoperatively.

In extreme cases, the impaction of food may occur, which can be seen as a radiolucent filling defect just proximal to the stoma. Immediate deflation of the cuff can be performed with a repeat study to document the free flow of barium through the increased caliber of the stoma.
2. Pouch dilatation with band slippage: Slippage of the band leads to a part of the stomach herniating superiorly and causes dilatation of the pouch. The incidence is approximately $24 \%$ and the band can slip anteriorly, posteriorly, or concentrically. It can further lead to complications like 

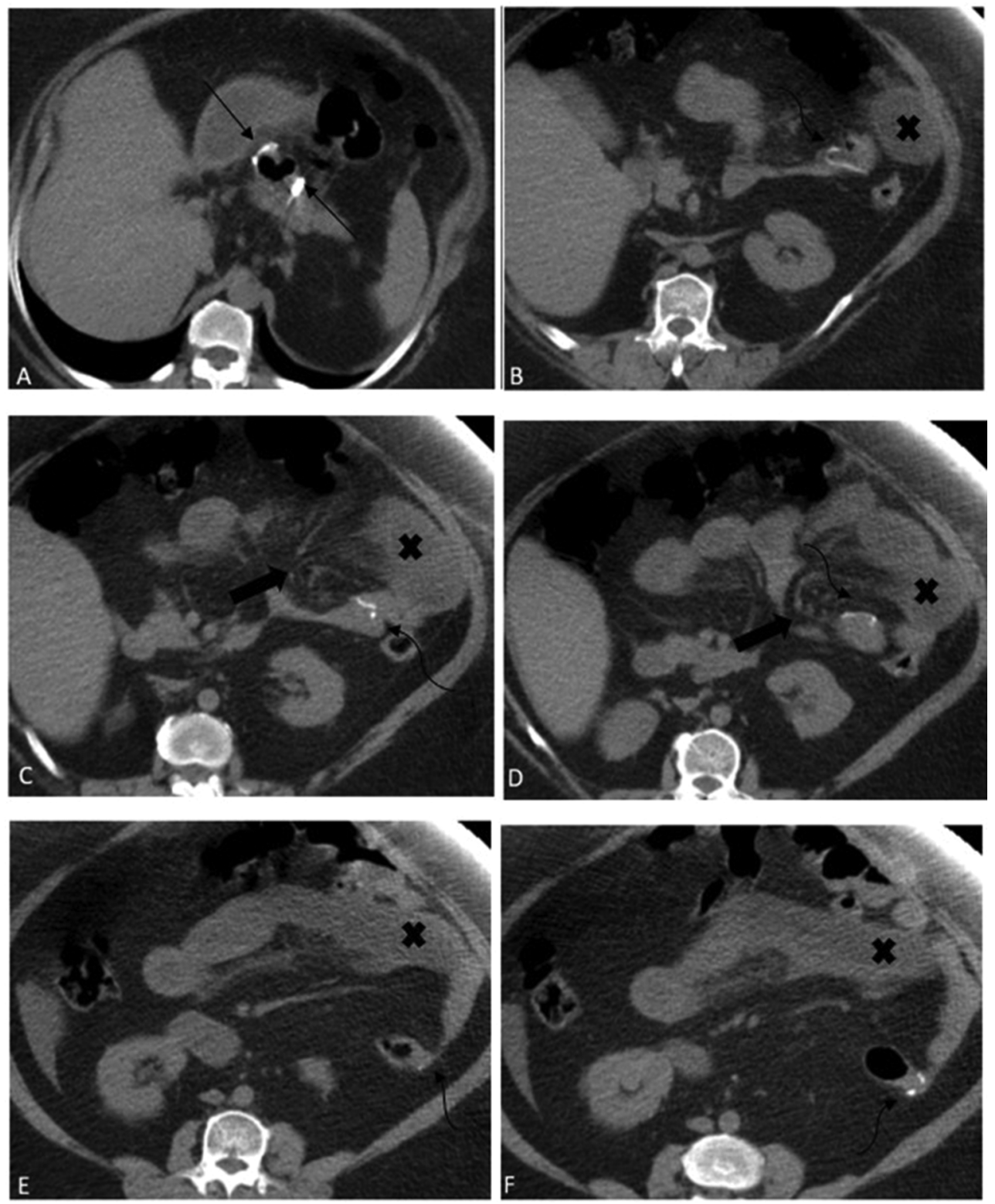

Fig. 16 Axial noncontrast computed tomography (A-F from cranial to caudal) in the same patient as in $\mathbf{- F i g . ~} \mathbf{1 5}$ showing gastrojejunal anastomosis (line arrow in $\mathbf{A}$ ) and jejunojejunal anastomosis (curved arrows in $\mathbf{B}-\mathbf{F})$. Clumped dilated small bowel loops (marked $\mathbf{X}$ in $\mathbf{B}-\mathbf{F})$ are seen with swirling of the mesenteric vessels (in $\mathbf{D}$ ) and radiating mesenteric vessels (in C) marked with block arrows suggestive of internal hernia.

acute gastric obstruction, gastric volvulus, ischemia, and infarction, leading to perforation; on diagnosis, immediate deflation of the band is required. In cases of anterior slippage, the anterior and superior portions of the distal stomach herniate, causing rotation of the band with an increased phi angle. Posterior slippage occurs in association with transbursal band placement and causes the posterior and inferior portions of the distal stomach to herniate. A newer technique with the placement of the band high in the hepatogastric ligament without violation of the lesser sac, so that the band is distant from the peristaltic stomach. In posterior slippage, the band is seen to have an O-shape as the anterior and posterior portions do not overlap ( - Fig. 20) ${ }^{43}$

3. Intragastric erosion: This is a delayed complication observed in 0.3 to $14 \%$ of patients. The various causes include small gastric wall injuries incurred during band placement, overdistension of the cuff leading to ischemia, band site infection, use of nonsteroidal anti-inflammatory drugs (NSAIDs), and so on. The gastric band may sink into a minor tear in the gastric wall and eventually erode through the lumen. A clinical sign of erosion would be the inability to inflate the port or recurrent port site infections, and they present with nonspecific abdominal pain, hematemesis or aspiration of turbid fluid from the port. On scout films, progressive displacement of the band as compared with prior films should raise the suspicion of erosion. Initial contrast studies are normal; at later stages, the contrast will be seen surrounding the eroded portion of the band. A definitive diagnosis can be made on CT when there is continuity between the band and the gastric lumen (opacified with dilute water-soluble 

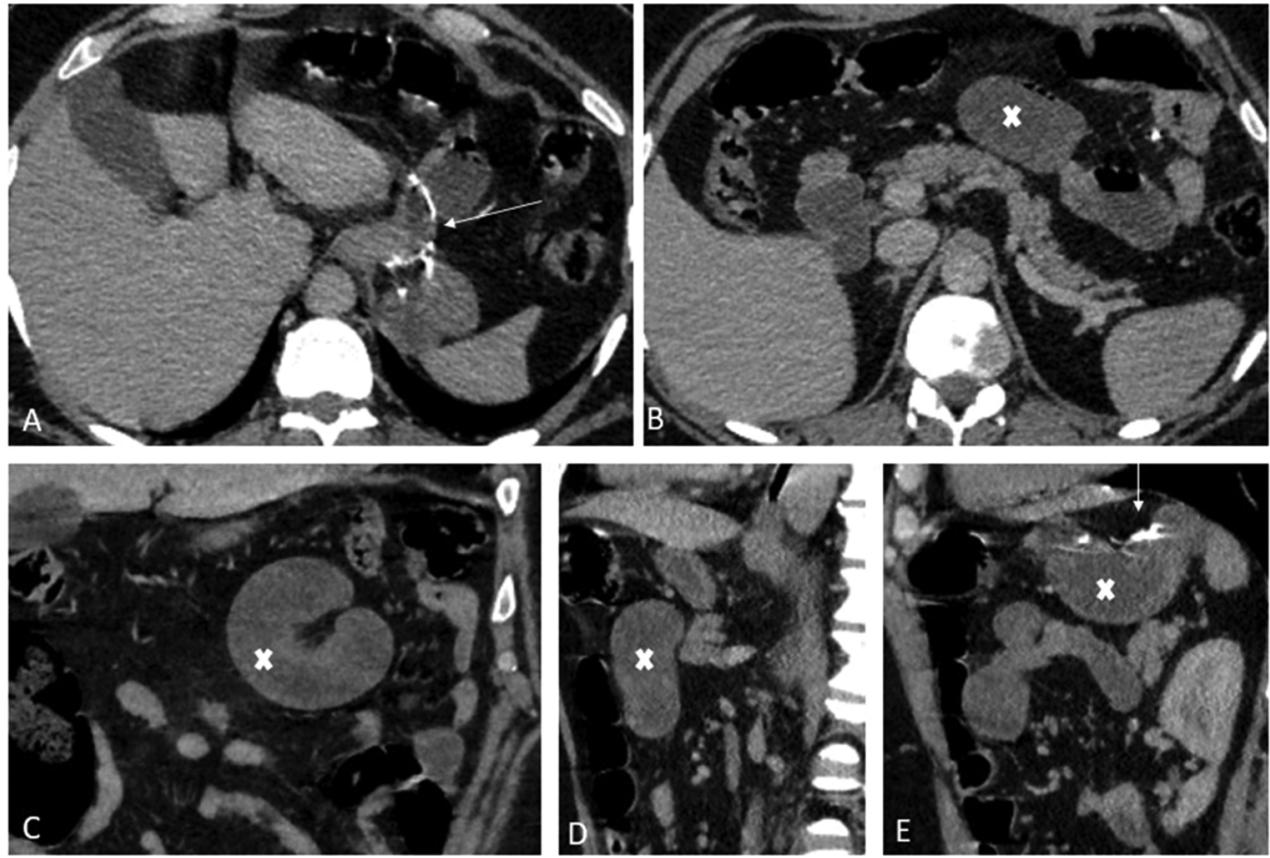

Fig. 17 Axial noncontrast computed tomography (A and B), coronal (C) and sagittal ( $\mathbf{D}$ and $\mathbf{E}$ ) in a patient 7 days post Roux-en-Y gastric bypass showing gastrojejunal anastomosis (line arrow in A). A closed loop of obstructed small bowel (marked $\mathbf{X}$ in $\mathbf{B}-\mathbf{E}$ ) is seen above the level of the transverse mesocolon in keeping with Peterson's hernia, which was confirmed intraoperatively.

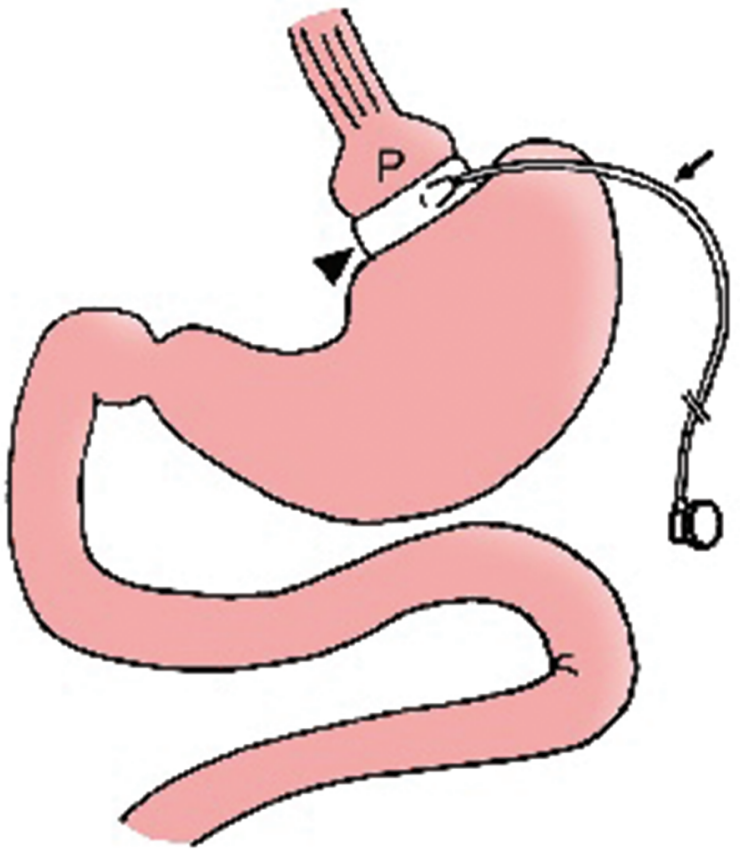

Fig. 18 Schematic representation of silicone band placed around the proximal stomach to create a pouch and narrow stoma communicating with the rest of the stomach. ${ }^{38}$

contrast) ${ }^{44}$ This warrants immediate removal of the band with a follow-up fluoroscopic examination within a week to rule out an ongoing leak.

4. Malpositioned band: Presence of the band in the perigastric fat that fails to encompass the stomach. It might also inadvertently be placed in the lower stomach, causing GOO. ${ }^{45}$

5. Esophageal widening and dysmotility: This usually occurs in the presence of an underlying insufficiency in the gastroesophageal sphincter, with inadequate dietary habit changes after surgery. It can lead to reflux, esophagitis, and aspiration. On upper GI study, the dilated esophagus is seen with reduced or absent peristalsis.

6. Port site-related and technical complications: Rotation and inversion of the injection port, tube disconnection, port site-related infections, and peritoneal adhesions leading to bowel obstruction can occur.

\section{Laparoscopic Mini Gastric Bypass}

This is a single anastomosis gastric bypass first described in 1990, and first performed laparoscopically in 2002.48 It was popularized in 2006 and after, and given the global trends, there is a suggestion that the uptake of laparoscopic mini gastric bypass (MGB) is significantly increasing. ${ }^{5} \mathrm{~A}$ meta-analysis published by Wang et al showed that MGB has several advantages over RYGB. These advantages are a higher 1 and 2-year excess weight loss, higher type II DM remission rate, and a shorter operation time. In terms of long-term results, MGB might be superior to laparoscopic sleeve gastrectomy (SG) also. ${ }^{49,50}$

Technique: A long gastric tube is created by dividing the stomach along the lesser curvature from the angle of His to a point approximately $2 \mathrm{~cm}$ proximal to the pylorus. The jejunum is divided approximately $200 \mathrm{~cm}$ distal to the ligament of Treitz, and a side-to-side anastomosis is made with the 

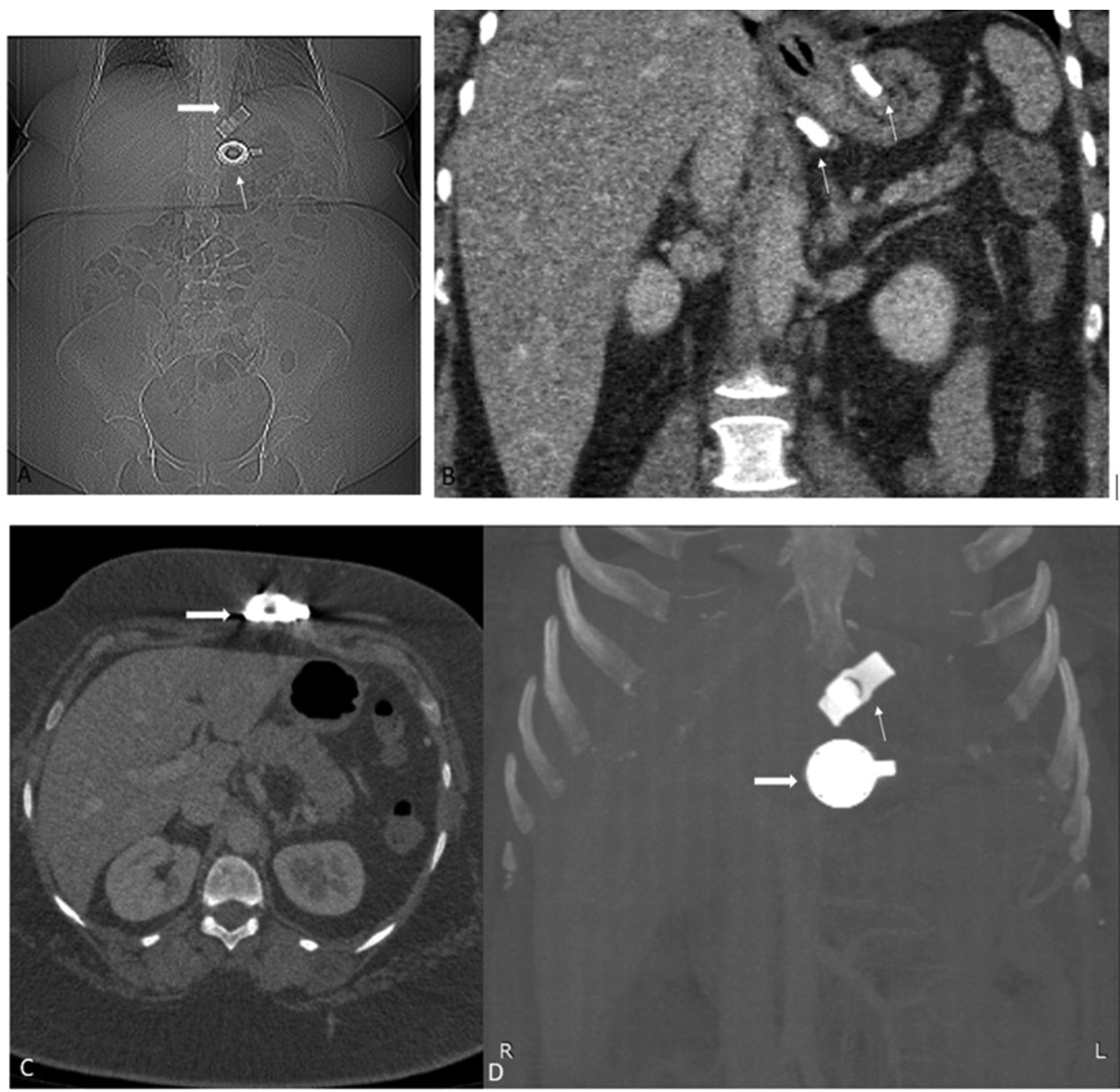

Fig. 19 Computed tomography (CT) scanogram (A) and coronal maximum intensity projection image (D) in a patient postgastric banding shows the normal appearance of the radiodense band (block arrow) and the subcutaneous port (line arrow). Coronal (B), axial (C) CT images show the gastric band around the proximal stomach (line arrow in B) with the port in the subcutaneous plane (block arrow in C).

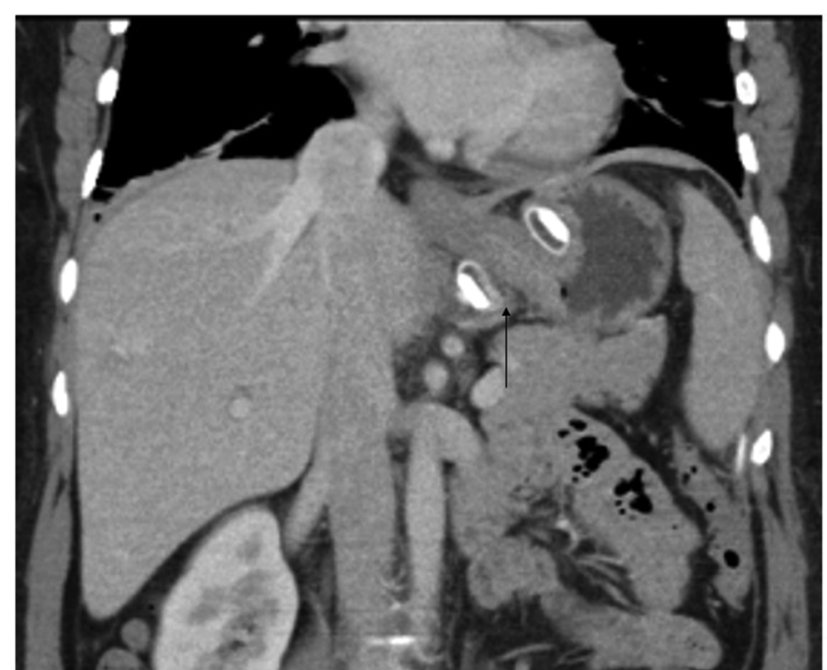

Fig. 20 Coronal contrast-enhanced computed tomography in a patient postgastric banding showing slippage of the band along the lesser curvature (line arrow), where it is not seen adherent to the gastric wall.

gastric tube to bypass the gastric antrum, duodenum, and proximal small bowel. The bowel is anastomosed antecolically at an acute angle to prevent bile reflux into the afferent loop ( - Fig. 21). . $^{51}$

\section{Normal Anatomy}

The tubular functional stomach and gastrojejunal anastomosis leading to the efferent limb is well visualized on upper GI study. CT can be used in suspicious cases where the radiodense surgical staples help identify the site of surgery. CT allows for visualization of the afferent limb as well (-Figs. 22-23).

\section{Complications}

The incidence of complications with MGB is found to be lesser than with RYGB. ${ }^{52}$ There are limited studies comparing it with SG, but the ones available are promising and show no significant difference between the two procedures. ${ }^{53,54} \mathrm{~A}$ study by Kansou et al showed MGB had better weight loss at 1 year compared with SG with higher gastric complications..$^{55}$ They include:

1. Anastomotic leak: The most common site is at the gastrojejunal anastomosis, and they can be diagnosed on upper GI study, as the presence of extraluminal contrast into the perigastric soft tissue. CT can be used to look for abscesses and subtle leaks. Pneumoperitoneum and localized fluid collection at unexpected sites are other signs of a leak on $\mathrm{CT}^{51}$ 


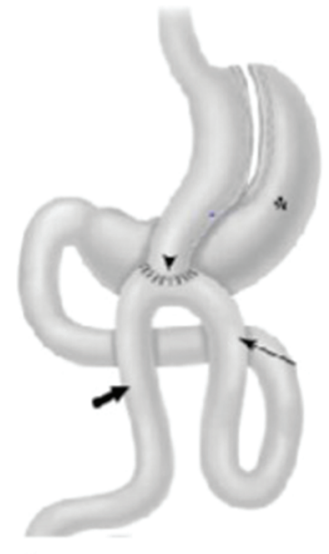

A

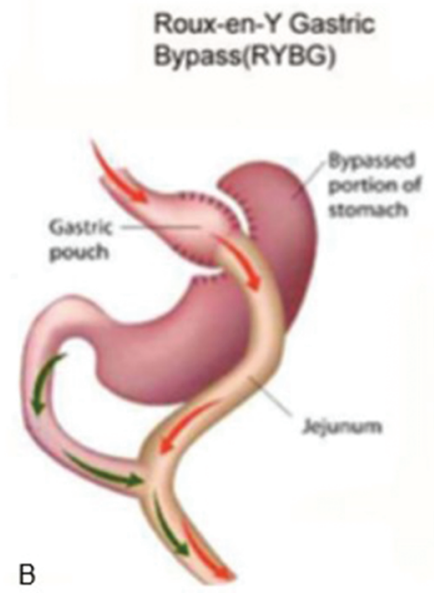

Mini Gastric Bypass

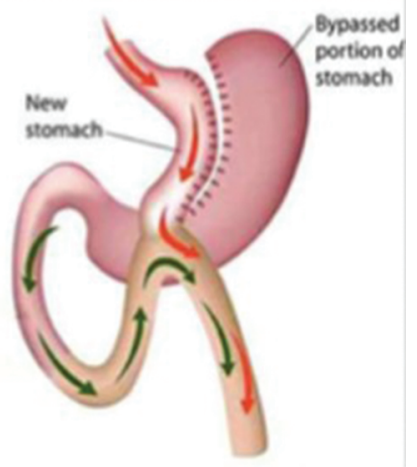

Fig. 21 (A) Schematic representation of mini gastric bypass (MGB) with the creation of a gastric tube and a side-to-side gastrojejunal anastomosis. ${ }^{25}$ (B) Shows the comparison between Roux-en-Y gastric bypass and MGB.
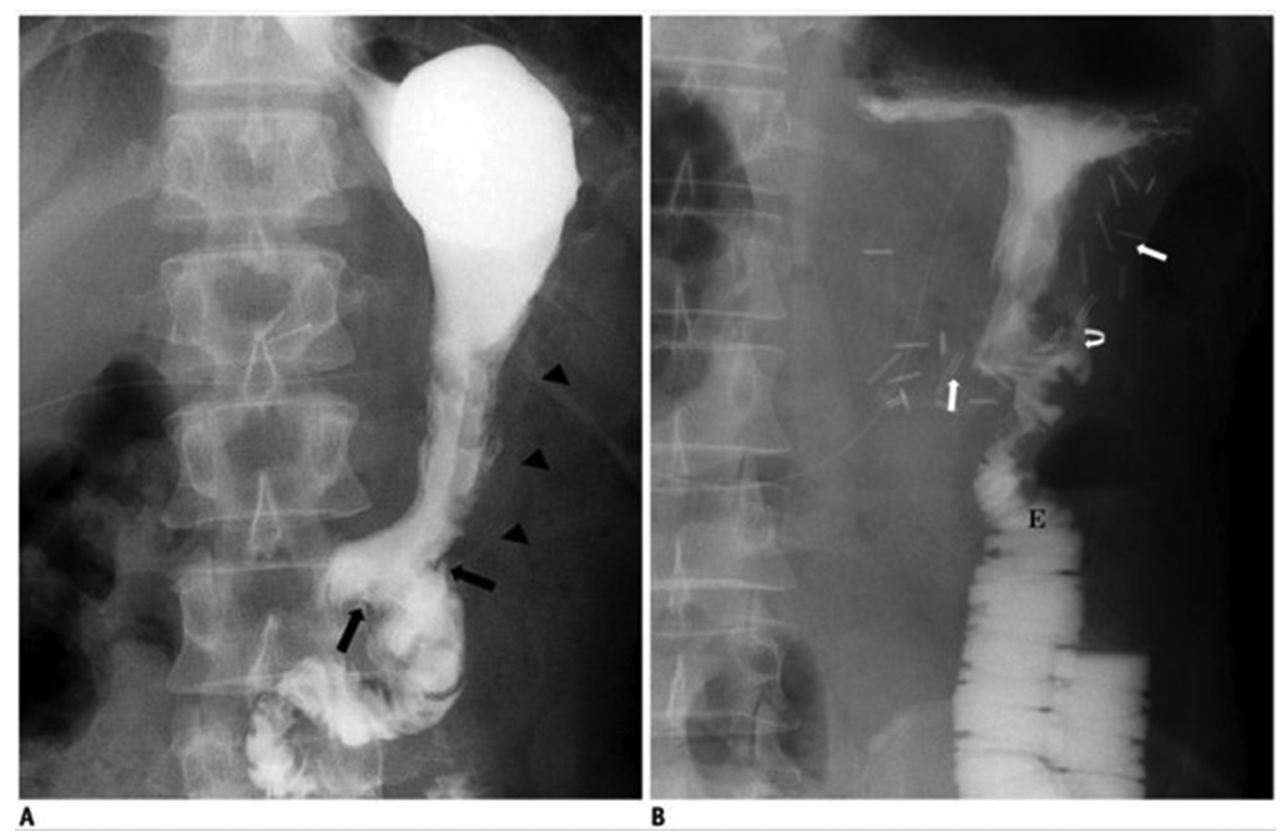

Fig. 22 Normal appearance of a patient postmini gastric bypass on upper gastrointestinal study with (A) showing side-to-side gastro-jejunostomy site (arrows) and faint staple lines (arrowheads) along greater curvature. (B). Afferent loop (curved arrow) partially seen with $\mathrm{E}$ representing the efferent loop. ${ }^{51}$

2. Stricture/stenosis: Early postoperative edema can lead to transient delayed emptying, which resolves with time. At later stages, stenosis at the anastomotic site is diagnosed as narrowing on upper GI studies and requires endoscopic balloon dilatation. ${ }^{25}$

3. Biliary reflux: This is the most notorious complication and is usually due to the presence of a large gastric tube. When severe, they may require surgery with stapling of the afferent limb just proximal to the gastrojejunal anastomosis and a laterolateral jejunojejunostomy..$^{56}$

4. Marginal ulcers: These are caused by large gastric tube and exposure to acid and are more reliably diagnosed on endoscopy, rather than upper GI study or CT.
5. Other complications include hemorrhage or hematoma at the operative site, wound-related complications like infection, and dehiscence. Anemia can be seen as a delayed complication due to bypassed duodenum. ${ }^{57}$

\section{Newer Bariatric Procedures}

Bridged MGB: This involves the creation of a $2 \mathrm{~cm}$ passage in the uppermost stomach that connects the relatively larger gastric pouch and the remnant stomach, in addition to the one anastomosis gastric bypass as described above. ${ }^{58}$ This has the following advantages as compared with RYGB and SG: 

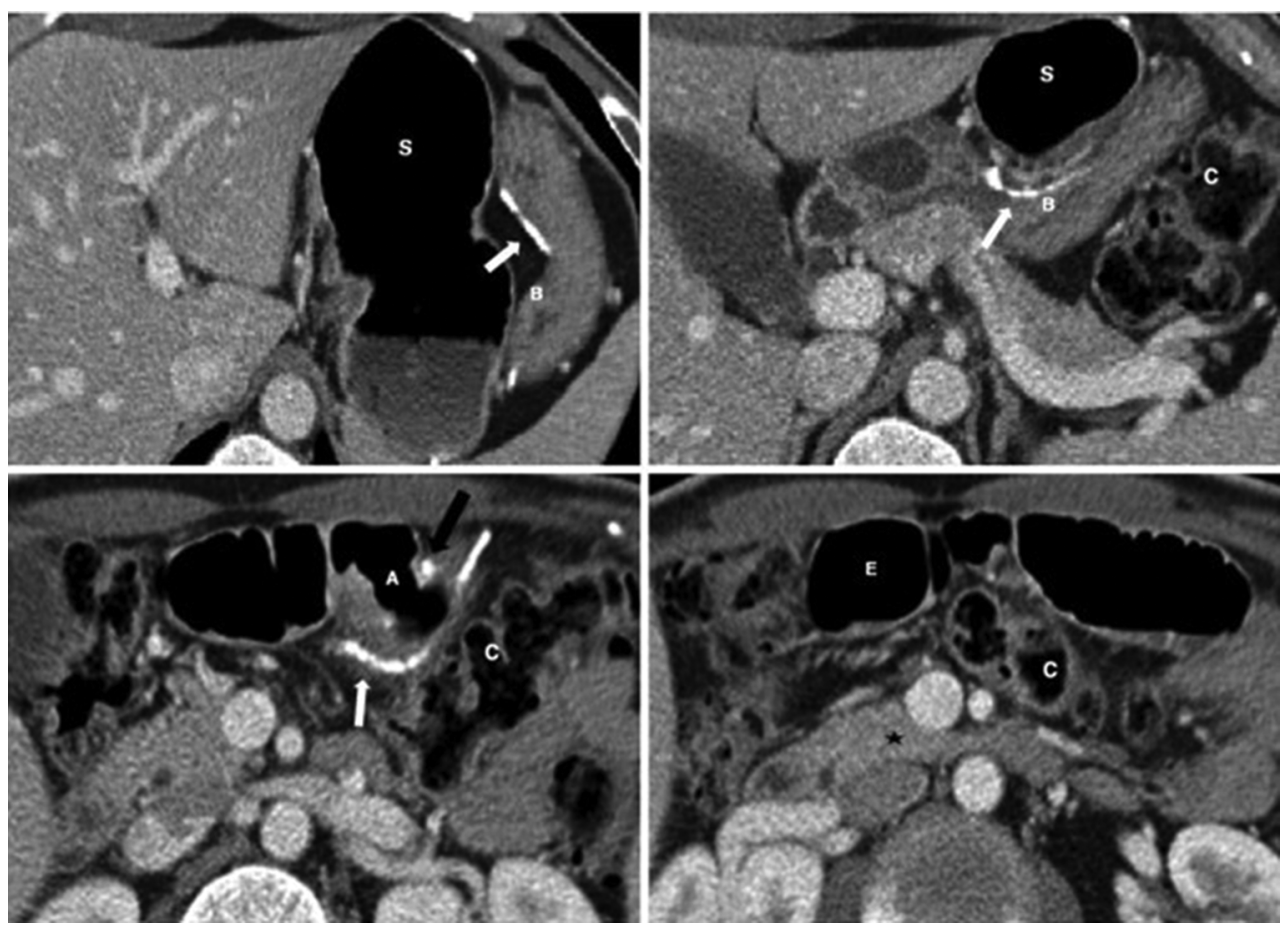

Fig. 23 Normal appearance in a patient post mini gastric bypass from cranial to caudal contrast-enhanced CT sections showing remnant stomach (S), bypassed segment (B), gastrojejunostomy (A) and distal loop (E). D3 segment of the duodenum $\left({ }^{*}\right)$ is a part of the afferent loop. Staple line along greater curvature of stomach (white arrows) and side-to-side gastrojejunostomy (black arrow). (C) Represents transverse colon. ${ }^{51}$

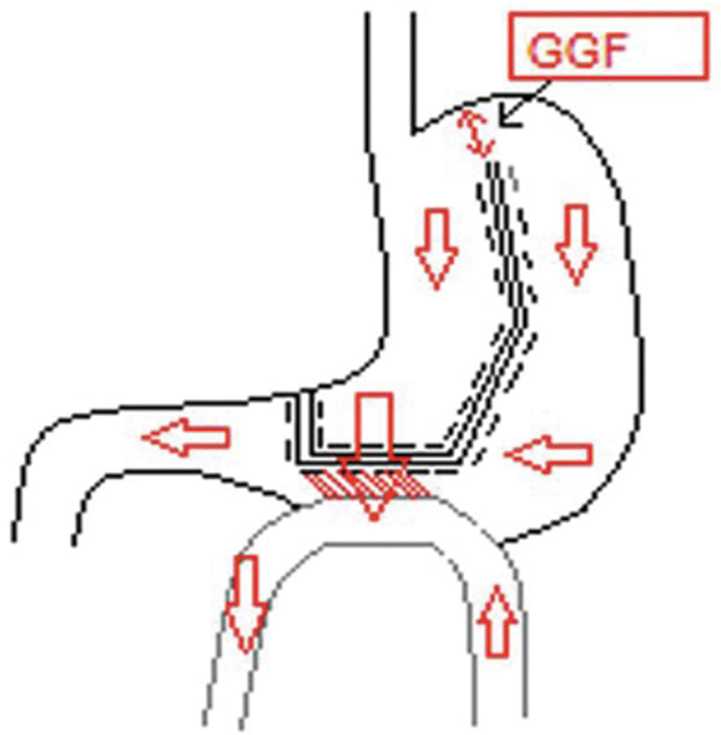

Fig. 24 Schematic representation showing the bridge at the most cranial $2 \mathrm{~cm}$ of fundus-artificial gastrogastric fistula (GGF) and the single gastrojejunal anastomosis (large red block arrow)..$^{59}$

1. The theoretical risk of a leak is lower due to the connection between the pouch and the remnant stomach.

2. Allows for endoscopic surveillance of the remnant stomach, to identify operative and early postoperative bleed, and screening for gastric cancers (- Fig. 24).$^{59}$
Transarterial embolization of the gastric fundus: This targets the endocrinal function of the fundus to suppress appetite. In the bariatric embolization of arteries for the treatment of obesity (BEAT) trial, the left gastric and gastroepiploic arteries were embolized using 300 to 500 micron microspheres with weight reduction at 12 months in 20 adults, with maximal appetite suppression at 1 month and peak weight loss occurring after 6 months. ${ }^{60,61}$

Intragastric ballooning (IGB): This is performed on patients with failed clinical treatment and who are at high risk for surgical procedures. It includes the endoscopic placement of a silicon intragastric balloon which is inflated with fluid or air during placement; it works by the principle of early satiety and delayed gastric emptying. ${ }^{62}$ Complications include spontaneous deflation, difficulty in removal and gastric ulcers. IGB treatment, in addition to lifestyle modification, is considered to be an effective short-term modality for weight loss in selected patients. However, there is insufficient evidence supporting its long-term efficacy ${ }^{63}$ (-Fig. 25).

\section{Conclusion}

Given the rise in the global incidence of obesity, bariatric surgical procedures are increasingly being performed. The knowledge regarding the type of surgery performed is important in identifying the potential complications in both the emergent and nonemergent setting. The radiologist needs to be familiar with the varying surgical trends and the contemporary as well as the more outdated obsolete procedures to make the right diagnosis. 


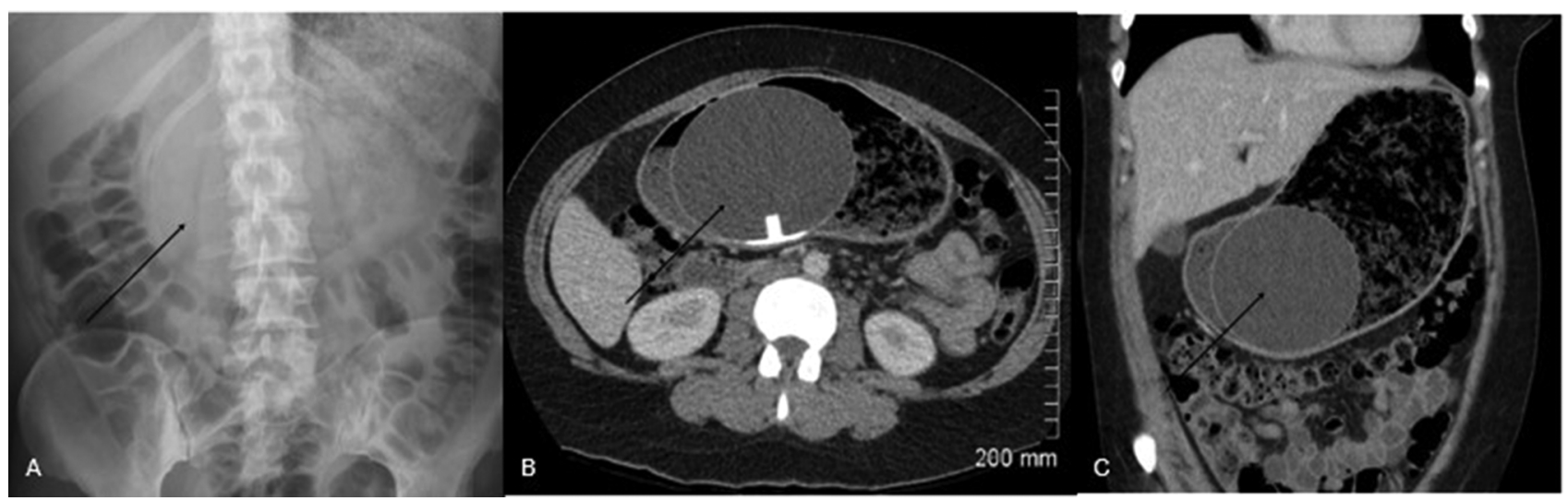

Fig. 25 Normal appearance of intragastric balloon (line arrow) on plain radiograph (A) of the abdomen as a well-defined density in the expected area of the stomach. Axial (B) and coronal (C) contrast-enhanced CT showing the gastric balloon (line arrow) in place. ${ }^{64}$

\section{Conflict of Interest}

None declared.

\section{Acknowledgments}

The authors would like to express their thanks to Dr. Sumit Talwar, Consultant Bariatric Surgeon, Manipal Hospital, Bangalore for his precious inputs and encouragement and Dr. Aruna. R Patil, Consultant Radiologist, Apollo Hospitals, Bannerghatta, Bangalore, for her help.

\section{References}

1 Bariatric surgery in India Evidence Summary Tool. Available at http://www.ifso-apc.com/pdf/bariatric-surgery-in-india-evidence-summary-tool.pdf. Accessed April 8, 2020.

2 Obesity and overweight. Available at: https://www.who. int/news-room/fact-sheets/detail/obesity-and-overweight. Accessed April 6, 2020.

3 Bhardwaj S, Misra A, Misra R, et al. High prevalence of abdominal, intra-abdominal and subcutaneous adiposity and clustering of risk factors among urban Asian Indians in North India. PLoS One 2011;6(9):e24362

4 Singh AK, Singh R, Kota SK. Bariatric surgery and diabetes remission: who would have thought it? Indian J Endocrinol Metab 2015;19(5):563-576

5 Ramos A, Fasmbs F, Kow L, et al. The IFSO Global Registry 5th IFSO Global Registry Report. Available at: https://www.ifso. com/pdf/5th-ifso-global-registry-report-september-2019.pdf. Accessed April 28, 2020

6 Benalcazar DA, Cascella M. Obesity surgery pre-op assessment and preparation. Available at: http://www.ncbi.nlm.nih.gov/ pubmed/31536273. Accessed April 29, 2020.

7 Schlottmann F, Buxhoeveden R. Laparoscopic Roux-en-Y gastric bypass: surgical technique and tips for success. J Laparoendosc Adv Surg Tech A 2018;28(8):938-943

8 Pescatori LC, Savarino E, Mauri G, et al. Quantification of visceral adipose tissue by computed tomography and magnetic resonance imaging: reproducibility and accuracy. Radiol Bras 2019;52(1):1-6

9 Torriani M, Oliveira AL, Azevedo DC, Bredella MA, Yu EW. Effects of Roux-en-Y gastric bypass surgery on visceral and subcutaneous fat density by computed tomography. Obes Surg 2015;25(2):381-385
10 Merlotti C, Ceriani V, Morabito A, Pontiroli AE. Subcutaneous fat loss is greater than visceral fat loss with diet and exercise, weight-loss promoting drugs and bariatric surgery: a critical review and meta-analysis. Int J Obes 2017;41(5):672-682

11 Gloy VL, Briel M, Bhatt DL, et al. Bariatric surgery versus non-surgical treatment for obesity: a systematic review and meta-analysis of randomised controlled trials. BMJ 2013;347:f5934-f5934

12 Wu F-Z, Huang Y-L, Wu CC, et al. Differential effects of bariatric surgery versus exercise on excessive visceral fat deposits. Medicine (Baltimore) 2016;95(5):e2616-e2616

13 Gumbs AA, Gagner M, Dakin G, Pomp A. Sleeve gastrectomy for morbid obesity. Obes Surg 2007;17(7):962-969

14 Levine MS, Carucci LR. Imaging of bariatric surgery: normal anatomy and postoperative complications. Radiology 2014;270(2):327-341

15 Riaz RM, Myers DT, Williams TR. Multidetector CT imaging of bariatric surgical complications: a pictorial review. Abdom Radiol (NY) 2016;41(1):174-188

16 Lehnert B, Moshiri M, Osman S, et al. Imaging of complications of common bariatric surgical procedures. Radiol Clin North Am 2014;52(5):1071-1086

17 Higa KD, Boone KB, Ho T, Davies OG. Laparoscopic Roux-en-Y gastric bypass for morbid obesity: technique and preliminary results of our first 400 patients. Arch Surg 2000;135(9):10291033, discussion 1033-1034

18 Schauer PR, Ikramuddin S, Gourash W, Ramanathan R, Luketich J. Outcomes after laparoscopic Roux-en-Y gastric bypass for morbid obesity. Ann Surg 2000;232(4):515-529

19 Fisher BL, Schauer P. Medical and surgical options in the treatment of severe obesity. Am J Surg 2002;184(6B) :9S-16S

20 DeMaria EJ, Sugerman HJ, Kellum JM, Meador JG, Wolfe LG. Results of 281 consecutive total laparoscopic Roux-en-Y gastric bypasses to treat morbid obesity. Ann Surg 2002;235(5):640645, discussion 645-647

21 Higa KD, Boone KB, Ho T. Complications of the laparoscopic Roux-en-Y gastric bypass: 1,040 patients-what have we learned? Obes Surg 2000;10(6):509-513

22 Al Harakeh AB, Kallies KJ, Borgert AJ, Kothari SN. Bowel obstruction rates in antecolic/antegastric versus retrocolic/ retrogastric Roux limb gastric bypass: a meta-analysis. Surg Obes Relat Dis 2016;12(1):194-198

23 Escalona A, Devaud N, Pérez G, et al. Antecolic versus retrocolic alimentary limb in laparoscopic Roux-en-Y gastric bypass: a comparative study. Surg Obes Relat Dis 2007;3(4):423-427

24 Aghajani E, Jacobsen HJ, Nergaard BJ, Hedenbro JL, Leifson BG, Gislason H. Internal hernia after gastric bypass: a new and 
simplified technique for laparoscopic primary closure of the mesenteric defects. J Gastrointest Surg 2012;16(3):641-645

25 Yazgan Ç, Balcı S, Özmen M. Post-operative imaging of baraitric surgery: What radiologists needs to know. EC Gastroenterol Dig Syst 2018;5(10):776-786

26 Ballesta C, Berindoague R, Cabrera M, Palau M, Gonzales M. Management of anastomotic leaks after laparoscopic Rouxen-Y gastric bypass. Obes Surg 2008;18(6):623-630

27 Fernanda S, Mazzariol MD, Ellen L, Wolf M. Bariatric/metabolic surgery for the radiologist-part 2: laparoscopic Roux-en-Y gastric bypass. Appl Radiol 2016;45(12):7-15

28 Blachar A, Federle MP. Gastrointestinal complications of laparoscopic roux-en-Y gastric bypass surgery in patients who are morbidly obese: findings on radiography and CT. AJR Am J Roentgenol 2002;179(6):1437-1442

29 Tucker ON, Escalante-Tattersfield T, Szomstein S, Rosenthal RJ. The ABC System: a simplified classification system for small bowel obstruction after laparoscopic Roux-en-Y gastric bypass. Obes Surg 2007;17(12):1549-1554

30 Higa KD, Ho T, Boone KB. Internal hernias after laparoscopic Roux-en-Y gastric bypass: incidence, treatment and prevention. Obes Surg 2003;13(3):350-354

31 Carucci LR, Turner MA, Shaylor SD. Internal hernia following Roux-en-Y gastric bypass surgery for morbid obesity: evaluation of radiographic findings at small-bowel examination. Radiology 2009;251(3):762-770

32 Dilauro M, McInnes MDF, Schieda N, et al. Internal hernia after laparoscopic Roux-en-Y gastric bypass: optimal CT Signs for diagnosis and clinical decision making. Radiology 2017;282(3):752-760

33 Lockhart ME, Tessler FN, Canon CL, et al. Internal hernia after gastric bypass: sensitivity and specificity of seven CT signs with surgical correlation and controls. AJR Am J Roentgenol 2007;188(3):745-750

34 Goodman P, Halpert RD. Radiological evaluation of gastric stapling procedures for morbid obesity. Crit Rev Diagn Imaging 1991;32(1):37-67

35 Hainaux B, Agneessens E, Rubesova E, et al. Intragastric band erosion after laparoscopic adjustable gastric banding for morbid obesity: imaging characteristics of an underreported complication. AJR Am J Roentgenol 2005;184(1):109-112

36 Vinzens F, Kilchenmann A, Zumstein V, Slawik M, Gebhart M, Peterli R. Long-term outcome of laparoscopic adjustable gastric banding (LAGB): results of a Swiss single-center study of 405 patients with up to 18 years' follow-up. Surg Obes Relat Dis 2017;13(8):1313-1319

37 Aarts EO, Dogan K, Koehestanie P, Aufenacker TJ, Janssen IMC, Berends FJ. Long-term results after laparoscopic adjustable gastric banding: a mean fourteen year follow-up study. Surg Obes Relat Dis 2014;10(4):633-640

38 Clayton RD, Carucci LR. Imaging following bariatric surgery: rouxen-Y gastric bypass, laparoscopic adjustable gastric banding and sleeve gastrectomy. Br J Radiol 2018;91(1089):20180031

39 Wiesner W, Schöb O, Hauser RS, Hauser M. Adjustable laparoscopic gastric banding in patients with morbid obesity: radiographic management, results, and postoperative complications. Radiology 2000;216(2):389-394

40 DeMaria EJ, Sugerman HJ, Meador JG, et al. High failure rate after laparoscopic adjustable silicone gastric banding for treatment of morbid obesity. Ann Surg 2001;233(6):809-818

41 Frigg A, Peterli R, Zynamon A, Lang C, Tondelli P. Radiologic and endoscopic evaluation for laparoscopic adjustable gastric banding: preoperative and follow-up. Obes Surg 2001;11(5):594-599

42 Chevallier J-M, Zinzindohoué F, Douard R, et al. Complications after laparoscopic adjustable gastric banding for morbid obesity: experience with 1,000 patients over 7 years. Obes Surg 2004;14(3):407-414

43 Pieroni S, Sommer EA, Hito R, Burch M, Tkacz JN. The "O" sign, a simple and helpful tool in the diagnosis of laparoscopic adjustable gastric band slippage. AJR Am J Roentgenol 2010;195(1):137-141

44 Sonavane SK, Menias CO, Kantawala KP, et al. Laparoscopic adjustable gastric banding: what radiologists need to know. Radiographics 2012;32(4):1161-1178

45 Prosch H, Tscherney R, Kriwanek S, Tscholakoff D. Radiographical imaging of the normal anatomy and complications after gastric banding. Br J Radiol 2008;81(969):753-757

46 O'Brien PE, Dixon JB. Lap-band: outcomes and results. J Laparoendosc Adv Surg Tech A 2003;13(4):265-270

47 Mortelé KJ, Pattijn P, Mollet P, et al. The Swedish laparoscopic adjustable gastric banding for morbid obesity: radiologic findings in 218 patients. AJR Am J Roentgenol 2001;177(1):77-84

48 Zakaria EA. Laparoscopic one-anastomosis gastric bypass: results of the first 310 patients. Egypt J Surg 2019;38(3):406

49 Wang F-G, Yu Z-P, Yan W-M, Yan M, Song M-M. Comparison of safety and effectiveness between laparoscopic mini-gastric bypass and laparoscopic sleeve gastrectomy: A meta-analysis and systematic review. Medicine (Baltimore) 2017;96(50):e8924-e8924

50 Carbajo M, García-Caballero M, Toledano M, Osorio D, García-Lanza C, Carmona JA. One-anastomosis gastric bypass by laparoscopy: results of the first 209 patients. Obes Surg 2005;15(3):398-404

51 Park HJ, Hong SS, Hwang J, Hur KY. Mini-gastric bypass to control morbid obesity and diabetes mellitus: what radiologists need to know. Korean J Radiol 2015;16(2):325-333

52 Lee WJ, Ser KH, Lee YC, Tsou JJ, Chen SC, Chen JC. Laparoscopic Roux-en-Y vs. mini-gastric bypass for the treatment of morbid obesity: a 10-year experience. Obes Surg 2012;22(12):1827-1834

53 Mostafa, Wahab, EMA, Sayed, YGA, Gafar, MH. Laparoscopic sleeve gastrectomy versus laparoscopic mini-gastric bypass in management of morbid obesity and its comorbidities. Menoufia Med J 2018;31(4):1181

54 Nabil T, Hussein A, Nabil A. Comparative study between the complications of laparoscopic sleeve gastrectomy and laparoscopic mini-gastric bypass. Egypt J Surg 2017;36(4):360

55 Kansou G, Lechaux D, Delarue J, et al. Laparoscopic sleeve gastrectomy versus laparoscopic mini gastric bypass: One year outcomes. Int J Surg 2016;33(Pt A) :18-22

56 Noun R, Skaff J, Riachi E, Daher R, Antoun NA, Nasr M. One thousand consecutive mini-gastric bypass: short- and longterm outcome. Obes Surg 2012;22(5):697-703

57 Kular KS, Manchanda N, Rutledge R. Analysis of the fiveyear outcomes of sleeve gastrectomy and mini gastric bypass: a report from the Indian sub-continent. Obes Surg 2014;24(10):1724-1728

58 Chaim EA, Ramos AC, Cazzo E. MINI-gastric bypass: description of the technique and preliminary results. Arq Bras Cir Dig 2017;30(4):264-266

59 Sumer A, Aktokmakyan TV, Peksen C. Mini gastric by-pass and bridged mini gastric by-pass: what is new? Available at: http://ales.amegroups.com/article/view/5599. Accessed January 7, 2020

60 Weiss CR, Akinwande O, Paudel K, et al. Clinical safety of bariatric arterial embolization: preliminary results of the BEAT obesity trial. Radiology 2017;283(2):598-608

61 Weiss CR, Abiola GO, Fischman AM, et al. bariatric embolization of arteries for the treatment of obesity (BEAT obesity) trial: results at 1 year. Radiology 2019;291(3):792-800

62 Fernandes M, Atallah ÁN, Soares BG, et al. Intragastric balloon for obesity. Cochrane Database Syst Rev 2007; (1):CD004931

63 Saber AA, Shoar S, Almadani MW, et al. Efficacy of first-time intragastric balloon in weight loss: a systematic review and meta-analysis of randomized controlled trials. Obes Surg 2017;27(2):277-287

64 Bariatric intragastric ballon. Available at: https://radiopaedia. org/cases/62114intragastric-ballon. Accessed October 19, 2020 